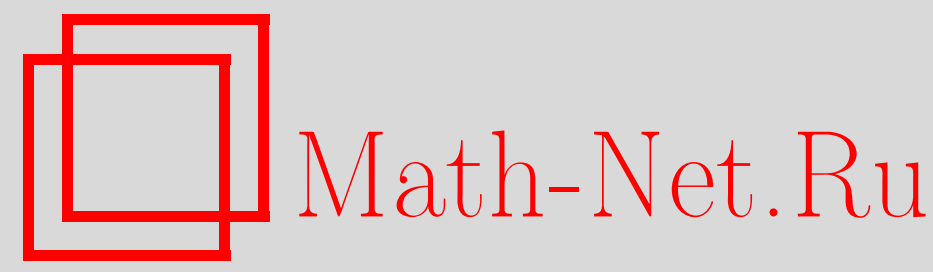

В. Н. Соловьев, О субдифференциале и производных по направлениям максимума семейства выпуклых функций, Изв. РАН. Сер. матем., 1998, том 62, выпуск 4, 173-200

DOI: https://doi.org/10.4213/im192

Использование Общероссийского математического портала Math-Net.Ru подразумевает, что вы прочитали и согласны с пользовательским соглашением

http://www.mathnet.ru/rus/agreement

Параметры загрузки:

IP : 54.197.130.99

26 апреля 2023 г., 16:38:00 
УДК 519.7

\title{
В.Н. Соловьев
}

\section{О субдифференциале и производных по направлениям максимума семейства выпуклых функций}

\author{
В работе вычисляются производные по направлениям и субдифференциалы \\ максимума выпуклых функций при ослабленных условиях компактности мно- \\ жества индексов (или их полном отсутствии). \\ Библиография: 30 наименований.
}

\section{§1. Введение}

В теории экстремальных задач и ее приложениях часто встречается и играет важную роль (см. [1]-[28]) задача вычисления производной по направлениям и субдифференциала функции $f(x)$, имеюшей вид

$$
f(x):=\sup _{s \in S} f_{s}(x)
$$

где $S$ - множество индексов, $f_{s}(x)$ - выпуклые функции, определенные на линейном пространстве $X$ (которые могут принимать значения $\pm \infty$ ). Поскольку функция $f(x)$ выпукла, то в любой точке $x_{0}$, в которой она конечна, существуют производные по направлениям $f^{\prime}\left(x_{0} ; \psi\right)$ (возможно, равные $\pm \infty$ ). В нескольких работах показано, что

$$
f^{\prime}\left(x_{0} ; \psi\right)=\sup _{s \in S\left(x_{0}\right)} f_{s}^{\prime}\left(x_{0} ; \psi\right)
$$

где

$$
S(x):=\left\{s \in S \mid f_{s}(x)=f(x)\right\} .
$$

При этом обычно предполагается, что $S$ - компактное подмножество топологического пространства $Y$ и функция $f(s, x):=f_{s}(x)$ непрерывна по совокупности аргументов на множестве $S \times\left[x_{0}-\varepsilon \psi, x_{0}+\varepsilon \psi\right], \varepsilon>0$ (см., например, [14]).

Для многих приложений эти требования слишком обременительны, и в данной работе они будут ослаблены. Напомним, что если $X$ - линейное топологическое пространство и $X^{*}$ - сопряженное пространство, т.е. множество всех линейных непрерывных функционалов $\left\langle x^{*}, x\right\rangle$ на $X$, то множество $\partial f\left(x_{0}\right):=\left\{x^{*} \in X^{*} \mid f(x) \geqslant\right.$ $\left.f\left(x_{0}\right)+\left\langle x^{*}, x-x_{0}\right\rangle \forall x \in X\right\}$ называется субдифференциалом функиии $f$ в точке $x_{0}$. Если $A$-некоторое подмножество пространства $X$, то множества

$$
\begin{aligned}
& N\left(x_{0} \mid A\right):=\left\{x^{*} \in X^{*} \mid\left\langle x^{*}, x-x_{0}\right\rangle \leqslant 0 \quad \forall x \in A\right\}, \\
& K\left(x_{0} \mid A\right):=\left\{\psi \in X \mid \exists \varepsilon>0: x_{0}+\varepsilon \psi \in A\right\}
\end{aligned}
$$

Работа выполнена при частичной поддержке РФФИ (грант № 96-01-00920).

$$
\text { (C) В.Н. Соловьев } 1998
$$


называются нормальным конусоми конусом допустимых направлений множества $A$ в точке $x_{0} \in A$ соответственно. Через $\operatorname{dom} f:=\{x: f(x)<+\infty\}$ обозначается эффективное множество функции $f(x)$. Легко проверить, что (см. [15, с. 263])

$$
\partial f\left(x_{0}\right)=\partial f\left(x_{0}\right)+N\left(x_{0} \mid \operatorname{dom} f\right) \quad \forall x_{0} \in \operatorname{dom} f .
$$

В дальнейшем всюду, не оговаривая этого, мы будем предполагать, что функции $f(s, x):=f_{s}(x): S \times X \rightarrow \overline{\mathbb{R}}:=\mathbb{R}^{1} \cup\{ \pm \infty\}$ выпуклы по $x \in X$ при каждом $s \in S$ и что $f(x) \neq-\infty$ при всех $x \in X$. Все топологии будут предполагаться хаусдорфовыми. Кроме того, всюду, кроме $\S 7$, мы будем предполагать, что

$$
\text { функиии } f(s, x) \text { полунепрерывны сверху по } s \in S \quad \forall x \in X \text {. }
$$

Будем назьвать функцию $f(s, x)$ коэрцитивной по $s$ в точке $x_{0} \in \operatorname{dom} f$, если

$$
\lim f\left(s, x_{0}\right)=-\infty \text { при } s \in S, \quad s \rightarrow s_{0} \notin S,
$$

когда множество $S$ не замкнуто, и если

$$
\lim f\left(s, x_{0}\right)=-\infty \text { при } s \in S, \quad\|s\| \rightarrow \infty
$$

когда оно не ограничено (и является подмножеством нормированного пространства $Y$; в условиях (1.6)-(1.8) множество $S$ снабжается относительной топологией, индуцируемой на $S$ из $Y$ ).

В $\S 2$ формула (1.2) будет доказана для полунепрерывных (пн.) сверху на множестве $S$ функций $f(\cdot, x)$ при ослабленных условиях компактности. При этом мы используем схему доказательства теоремы о субдифференциале максимума выпукльх функций из монографии [4]. С ее помощью в 33,4 получены усиления теорем о субдифференциале максимума семейств выпуклых функций, полученных А. Д. Иоффе, В.М. Тихомировым [4] и В.Л. Левиным [9], [10]. В 44 эти формулы упрощаются в тех случаях, когда функции $f(s, x)$ вогнуты по $s$. Здесь же даны интегральные представления субдифференциалов. В $\S 5$ изучены формулы для замыканий неограниченных выпуклых множеств в конечномерном пространстве, которые затем применяются в $\S 6$ при вычислении субдифференциалов в конечномерном случае. В $\S 7$ рассмотрен случай произвольного множества индексов $S$, а в $\S 8$ доказаны вспомогательные утверждения.

Основные результаты этой работы были анонсированы в [18], [19].

\section{§ 2. Производные по направлениям максимума выпуклых функций}

TеОрема 2.1. Пусть $X$ - линейное пространство, множсество $S$ компактно и выполнено условие (1.6). Тогда формула (1.2) справедлива для всех точек $x_{0} \in \operatorname{dom} f$ и допустимых направлений $\psi \in K\left(x_{0} \mid \operatorname{dom} f\right)$. Если же $\psi \notin K\left(x_{0} \mid \operatorname{dom} f\right)$, то найдется элемент $s \in S$ такой, что $f^{\prime}\left(x_{0} ; \psi\right)=$ $f_{s}^{\prime}\left(x_{0} ; \psi\right)=+\infty$. 
ДОКАЗАТЕЛЬСТВО. Фиксируем точку $x \in X$. Возможны три случая:

a) $f\left(s_{1}, x\right)=+\infty$ для некоторого $s_{1} \in S$; тогда $f(x)=+\infty$;

б) $f(s, x)=-\infty$ для всех $s \in S$; тогда $f(x)=-\infty$, что исключено по условию;

в) $f(s, x)<+\infty$ для всех $s \in S$ и $f\left(s_{1}, x\right)>-\infty$ для некоторого $s_{1} \in S$; тогда $f(x)$ конечно.

Утверждения а) и б) очевидны. В случае в) для числа $\gamma:=f\left(s_{1}, x\right)$ множество уровня

$$
S_{\gamma}:=\{s \in S \mid f(s, x) \geqslant \gamma\}
$$

будет непустым и замкнутым (по определению полунепрерывности сверху) подмножеством компактного топологического пространства $S$. Следовательно, множество $S_{\gamma}$ само компактно, и по теореме Вейерштрасса пн. сверху вешественная функция $f(\cdot, x): S_{\gamma} \rightarrow \mathbb{R}$ достигает на нем конечного максимума $f(x)$. В частности, поскольку по условию число $f\left(x_{0}\right)$ конечно, то множество $S\left(x_{0}\right)$, определенное в (1.3), является непустым компактом.

Легко показать, что [14]

$$
f^{\prime}\left(x_{0} ; \psi\right) \geqslant \sup _{s \in S\left(x_{0}\right)} f_{s}^{\prime}\left(x_{0} ; \psi\right) .
$$

Если $f^{\prime}\left(x_{0} ; \psi\right)=-\infty$, то и стоящая в правой части этого неравенства величина равна $-\infty$. Формула (1.2) доказана. Если же $f^{\prime}\left(x_{0} ; \psi\right)=+\infty$, то из выпуклости функции $f(x)$ и конечности ее значения в точке $x_{0}$ следует, что $f\left(x_{0}+\lambda \psi\right)=+\infty$, $\lambda>0$, т.е. $\psi \notin K\left(x_{0} \mid \operatorname{dom} f\right)$. Поэтому для любого $\lambda>0$ найдется элемент $s(\lambda) \in S$ такой, что $f\left(s(\lambda), x_{0}+\lambda \psi\right)=+\infty$. Действительно, как мы показали, в случае б) $f\left(x_{0}+\lambda \psi\right)=-\infty$, а в случае в) число $f\left(x_{0}+\lambda \psi\right)$ конечно.

Обозначим через $S(\lambda)$ множество всех таких элементов:

$$
S(\lambda):=\left\{s \in S \mid f\left(s, x_{0}+\lambda \psi\right)=+\infty\right\} \neq \varnothing, \quad \lambda>0 .
$$

Из выпуклости функции $f(s, x)$ по переменной $x$ и неравенств $f\left(s, x_{0}\right)<+\infty, s \in$ $S$, следует, что

$$
f\left(s, x_{0}+\mu \psi\right)=+\infty, \quad \mu \geqslant \lambda, \quad s \in S(\lambda) .
$$

Следовательно, $S\left(\lambda_{1}\right) \subset S\left(\lambda_{2}\right)$ при $\lambda_{1} \leqslant \lambda_{2}$. Кроме того, множество $S(\lambda)=$ $\bigcap_{\gamma \in \mathbb{R}}\left\{s \in S \mid f\left(s, x_{0}+\lambda \psi\right) \geqslant \gamma\right\}$ является пересечением компактных множеств уровня функции $f\left(\cdot, x_{0}+\lambda \psi\right)$, и поэтому само компактно. Таким образом, семейство множеств $S(\lambda), \lambda>0$, образует центрированную систему. Следовательно, пересечение этих множеств не пусто [8].

Согласно (2.3) это означает, что найдется элемент $s_{0} \in S$ такой, что $f\left(s_{0}, x_{0}+\right.$ $\mu \psi)=+\infty, \mu>0$, и поэтому $f_{s_{0}}^{\prime}\left(x_{0} ; \psi\right)=+\infty$.

Пусть теперь производная $f^{\prime}\left(x_{0} ; \psi\right)$ конечна. Тогда

$$
f\left(x_{0}+\lambda \psi\right) \in \mathbb{R}, \quad 0 \leqslant \lambda \leqslant \varepsilon,
$$

для некоторого $\varepsilon>0$, причем можно считать $\varepsilon$ равным 1 (этого можно добиться заменой вектора $\psi$ на $\varepsilon \psi$ ). Отметим сразу же, что

$$
\varliminf_{\lambda \rightarrow+0} f\left(x_{0}+\lambda \psi\right)=f\left(x_{0}\right)
$$


(в противном случае $f^{\prime}\left(x_{0} ; \psi\right)=-\infty$ в силу выпуклости функции $f(x)$ и конечности ее значения в точке $\left.x_{0}\right)$.

Выберем любые элементы $s(\lambda) \in S$, для которых

$$
f\left(s(\lambda), x_{0}+\lambda \psi\right) \geqslant f\left(x_{0}+\lambda \psi\right)-\lambda^{2}, \quad \lambda \in(0,1) .
$$

В силу выпуклости функции $f(s, x)$ по $x$ имеем

$$
(1-\lambda) f\left(s(\lambda), x_{0}\right)+\lambda f\left(s(\lambda), x_{0}+\psi\right) \geqslant f\left(s(\lambda), x_{0}+\lambda \psi\right),
$$

поэтому согласно (2.6)

$$
\begin{aligned}
(1-\lambda) f\left(s(\lambda), x_{0}\right) & \geqslant f\left(x_{0}+\lambda \psi\right)-\lambda^{2}-\lambda f\left(s(\lambda), x_{0}+\psi\right) \\
& \geqslant f\left(x_{0}+\lambda \psi\right)-\lambda^{2}-\lambda f\left(x_{0}+\psi\right) .
\end{aligned}
$$

Поскольку $f\left(x_{0}+\psi\right)$ кончено, то с учетом $(2.5)$ отсюда получаем

$$
\varliminf_{\lambda \rightarrow+0} f\left(s(\lambda), x_{0}\right) \geqslant f\left(x_{0}\right) .
$$

Ho $f\left(s(\lambda), x_{0}\right) \leqslant f\left(x_{0}\right)$, поэтому

$$
\lim _{\lambda \rightarrow+0} f\left(s(\lambda), x_{0}\right)=f\left(x_{0}\right) .
$$

В силу компактности множества $S$ из направленности $s(\lambda), 1>\lambda>0$, в которой индекс $\lambda$ направлен по убыванию, можно выделить поднаправленность $s(\lambda(\alpha))$, $\alpha \in A$, сходящуюся к элементу $s_{0} \in S$. Тогда из полунепрерывности сверху функции $f\left(\cdot, x_{0}\right)$ в точке $s_{0}$ и соотношения $(2.7)$ следует, что

$$
f\left(s(\lambda(\alpha)), x_{0}\right) \underset{A}{\longrightarrow} f\left(x_{0}\right) \leqslant f\left(s_{0}, x_{0}\right)
$$

Но в силу (1.1) очевидно обратное неравенство, поэтому

$$
f\left(s_{0}, x_{0}\right)=f\left(x_{0}\right),
$$

т.e. $s_{0} \in S\left(x_{0}\right)$.

Предположим теперь, что фиормула (1.2) не верна. Тогда неравенство (2.2) будет строгим и, в частности,

$$
f^{\prime}\left(x_{0} ; \psi\right) \geqslant f_{s_{0}}^{\prime}\left(x_{0} ; \psi\right)+\varepsilon
$$

для некоторого $\varepsilon>0$. Если производная $f_{s_{0}}^{\prime}\left(x_{0} ; \psi\right)$ конечна, то для некоторого числа $\lambda_{1} \in(0,1)$ будет

$$
\frac{\left[f\left(s_{0}, x_{0}+\lambda_{1} \psi\right)-f\left(s_{0}, x_{0}\right)\right]}{\lambda_{1}} \leqslant f_{s_{0}}^{\prime}\left(x_{0} ; \psi\right)+\frac{\varepsilon}{4},
$$


и поэтому в силу (2.9)

$$
f^{\prime}\left(x_{0} ; \psi\right) \geqslant \frac{\left[f\left(s_{0}, x_{0}+\lambda_{1} \psi\right)-f\left(s_{0}, x_{0}\right)\right]}{\lambda_{1}}+\frac{3 \varepsilon}{4}
$$

Иначе $f_{s_{0}}^{\prime}\left(x_{0} ; \psi\right)=-\infty$, и неравенство (2.10) тоже будет выполняться для некоторого числа $\lambda_{1} \in(0,1)$.

С учетом (2.6), (2.10) и выпуклости $f(x)$ в обоих случаях

$$
\begin{aligned}
& \frac{\left[f\left(s(\lambda), x_{0}+\lambda \psi\right)-f\left(s(\lambda), x_{0}\right)\right]}{\lambda} \geqslant \frac{\left[f\left(x_{0}+\lambda \psi\right)-\lambda^{2}-f\left(x_{0}\right)\right]}{\lambda} \\
& \geqslant f^{\prime}\left(x_{0} ; \psi\right)-\lambda \geqslant \frac{\left[f\left(s_{0}, x_{0}+\lambda_{1} \psi\right)-f\left(s_{0}, x_{0}\right)\right]}{\lambda_{1}}+\frac{3 \varepsilon}{4}-\lambda .
\end{aligned}
$$

Поэтому при $0<\lambda<\lambda_{1}$ в силу выпуклости функции $f(s(\lambda), x)$ по $x$ имеем

$$
\begin{aligned}
\frac{\lambda}{\lambda_{1}} f\left(s(\lambda), x_{0}+\lambda_{1} \psi\right) & +\left(1-\frac{\lambda}{\lambda_{1}}\right) f\left(s(\lambda), x_{0}\right) \geqslant f\left(s(\lambda), x_{0}+\lambda \psi\right) \\
\geqslant & f\left(s(\lambda), x_{0}\right)+\lambda\left(\frac{1}{\lambda_{1}}\left[f\left(s_{0}, x_{0}+\lambda_{1} \psi\right)-f\left(s_{0}, x_{0}\right)\right]+\frac{3 \varepsilon}{4}-\lambda\right),
\end{aligned}
$$

или, после сокращений,

$$
f\left(s(\lambda), x_{0}+\lambda_{1} \psi\right) \geqslant f\left(s(\lambda), x_{0}\right)+f\left(s_{0}, x_{0}+\lambda_{1} \psi\right)-f\left(s_{0}, x_{0}\right)+\left(\frac{3 \varepsilon}{4}-\lambda\right) \lambda_{1} .
$$

Но из (2.7) и (2.8) следует, что найдется число $0<\lambda_{2}<\min \left(\lambda_{1}, \varepsilon / 4\right)$ такое, что $f\left(s(\lambda), x_{0}\right) \geqslant f\left(s_{0}, x_{0}\right)-\varepsilon \lambda_{1} / 4,0<\lambda \leqslant \lambda_{2}$, и поэтому

$$
f\left(s(\lambda), x_{0}+\lambda_{1} \psi\right) \geqslant f\left(s_{0}, x_{0}+\lambda_{1} \psi\right)+\frac{\varepsilon \lambda_{1}}{4}, \quad 0<\lambda \leqslant \lambda_{2}
$$

Поскольку найдется такое $\alpha_{1} \in A$, что $0<\lambda(\alpha) \leqslant \lambda_{2}$ при всех $\alpha$, бо́льших $\alpha_{1}$ (по порядку) и $s(\lambda(\alpha)) \rightarrow s_{0}$, то полученное неравенство противоречит полунепрерывности сверху функции $f\left(\cdot, x_{0}+\lambda_{1} \psi\right)$ в точке $s_{0} \in S$. Теорема доказана.

ЗАМЕЧАНИЕ 2.1. В условиях теоремы 2.1 справедливо равенство

$$
K\left(x_{0} \mid \operatorname{dom} f\right)=\bigcap_{s \in S} K\left(x_{0} \mid \operatorname{dom} f_{s}\right)
$$

ТЕОРЕМА 2.2. Пусть $X$ - линейное пространство, многозначное отображение $S(x): \operatorname{dom} f \rightarrow S$ имеет непустые компактные значения и пн. сверху на любом отрезке в $\operatorname{dom} f$, а функиия $f(\cdot, x)$ пн. сверху на $S$ для любого $x \in X$. Тогда формула (1.2) справедлива для всех точек $x_{0} \in \operatorname{dom} f$ и направлений $\psi \in K\left(x_{0} \mid \operatorname{dom} f\right)$. 
ДокАЗАТЕЛЬСТво. Поскольку $\psi \in K\left(x_{0} \mid \operatorname{dom} f\right)$, то $f^{\prime}\left(x_{0} ; \psi\right) \neq+\infty$. Как мы видели, случай $f^{\prime}\left(x_{0} ; \psi\right)=-\infty$ тривиален. Поэтому остается рассмотреть случай, когда производная по направлению $f^{\prime}\left(x_{0} ; \psi\right)$ конечна. Тогда опять будут вьполняться соотношения (2.4), (2.5), а элементы $s(\lambda) \in S\left(x_{0}+\lambda \psi\right) \subset S$ будут удовлетворять условию (2.6). Как и в теореме 2.1 будет также выполнено ключевое соотношение (2.7).

Определим множество $\tilde{S}$, равное объединению множеств $S\left(x_{0}+\lambda \psi\right), \lambda \in[0,1]$. Так как отображение $S\left(x_{0}+\lambda \psi\right):[0,1] \rightarrow S$ имеет непустые компактные значения пн. сверху, то множество $\tilde{S}$ компактно (см. [13, с. 119]). Поэтому из направленности $s(\lambda) \in \tilde{S}, 1 \geqslant \lambda \geqslant 0$, в которой индекс $\lambda$ направлен по убыванию, можно выбрать поднаправленность $s(\lambda(\alpha)), \alpha \in A$, сходящуюся к элементу $s_{0} \in \tilde{S} \subset S$. Тогда, как и в теореме 2.1 , мы получим соотношения (2.8)-(2.11), последнее из которых противоречит полунепрерывности сверху функции $f\left(\cdot, x_{0}+\lambda_{1} \psi\right)$ в точке $s_{0} \in S$. Теорема доказана.

Tеорема 2.3. Пусть $X$ - линейное пространство, множество $S$ относительно компактно в $Y$, а функиия $f(\cdot, x)$ коэрцитивна в точке $x_{0} \in \operatorname{dom} f$ и пн. сверху на $S$ для любого $x \in X$. Тогда формула (1.2) справедлива для любого направления $\psi \in K\left(x_{0} \mid \operatorname{dom} f\right)$.

ДокаЗАТЕЛЬСТво. Поскольку $f\left(x_{0}\right) \in \mathbb{R}$, то $f\left(s, x_{0}\right)<+\infty$ при всех $s \in S$ и $f\left(s_{1}, x_{0}\right) \in \mathbb{R}$ для некоторого $s_{1} \in S$. Доопределим функцию $f(s, x)$ в точке $x=x_{0}$ на все пространство $Y$, положив

$$
f\left(s, x_{0}\right)=-\infty, \quad s \notin S .
$$

Легко сообразить, что тогда в силу условия (1.6), (1.7) функция

$$
f\left(\cdot, x_{0}\right): Y \rightarrow \mathbb{R} \cup\{-\infty\}
$$

будет полунепрерывной сверху в исходной топологии $Y$.

Поэтому при $\gamma=f\left(s_{1}, x_{0}\right)$ множество уровня

$$
\left\{s \in Y \mid f\left(s, x_{0}\right) \geqslant \gamma\right\}=S_{\gamma}
$$

будет непусто и замкнуто (см. (2.1)). Поскольку $S_{\gamma} \subset S$ и по условию замыкание $\bar{S}$ множества $S$ компактно, то множество $S_{\gamma}$ тоже компактно. Тогда при $x=x_{0}$ можно заменить множество $S$ в (1.1) на компактное множество $S_{\gamma}$. По теореме Вейерштрасса максимум в (1.1) достигается на непустом компактном подмножестве $S\left(x_{0}\right) \subset S_{\gamma} \subset S$.

Поскольку $\psi \in K\left(x_{0} \mid \operatorname{dom} f\right)$, то $f^{\prime}\left(x_{0} ; \psi\right) \neq+\infty$. Как мы видели, случай $f^{\prime}\left(x_{0} ; \psi\right)=-\infty$ тривиален. Поэтому остается рассмотреть случай, когда производная по направлению $f^{\prime}\left(x_{0} ; \psi\right)$ конечна. Тогда опять будут выполняться соотношения $(2.4),(2.5)$, и непосредственно из определения верхней грани (1.1) можно выбрать элементы $s(\lambda) \in S$, удовлетворяюшие условию (2.6). Как и в теореме 2.1 , они будут удовлетворять ключевому соотношению (2.7).

Пользуясь компактностью множества $\bar{S}$, из направленности $s(\lambda), 1 \geqslant \lambda \geqslant 0$, в которой индекс $\lambda$ направлен по убыванию, можно выбрать поднаправленность 
$s(\lambda(\alpha)), \alpha \in A$, сходящуюся к элементу $s_{0} \in Y$. Если $s_{0} \notin S$, то согласно условию (1.7)

$$
f\left(s(\lambda(\alpha)), x_{0}\right) \underset{A}{\longrightarrow}-\infty,
$$

что противоречит $(2.7)$, ибо число $f\left(x_{0}\right)$ конечно. Поэтому $s_{0} \in S$. Тогда, как и в теореме 2.1, мы получим соотношения (2.8)-(2.11), последнее из которых противоречит полунепрерывности сверху функции $f\left(\cdot, x_{0}+\lambda_{1} \psi\right)$ в точке $s_{0} \in S$. Теорема доказана.

ТеОРема 2.4. Пусть $X$ - линейное пространство, $S$ - подмножсество конечномерного пространства $Y=\mathbb{R}^{m}$, а функция $f(\cdot, x)$ коэриитивна в точке $x_{0} \in \operatorname{dom} f$ и пн. сверху на множсестве $S$ при всех $x \in X$. Тогда формула (1.2) справедлива для всех $\psi \in K\left(x_{0} \mid \operatorname{dom} f\right)$.

ДокаЗАТЕЛЬСтво. Как и в теореме 2.3, множества уровня функции (2.14), (2.13) будут замкнуты, а из условия (1.8) следует их ограниченность. Поэтому в конечномерном пространстве множества уровня (2.1) при $x=x_{0}$ компактны. Следовательно, множество (1.3) является непустым компактом.

Когда производная по направлению $f^{\prime}\left(x_{0} ; \psi\right)$ конечна, будут выполнены соотношения (2.4)-(2.7). Если бы последовательность $s_{n}:=s(1 / n), n=1,2, \ldots$, была неограниченной, то согласно (1.8) мы получили бы, что $f\left(s_{n}, x_{0}\right) \rightarrow-\infty$ при $n \rightarrow \infty$, что противоречит $(2.7)$, ибо число $f\left(x_{0}\right)$ конечно. Поэтому существует сходящаяся подпоследовательность $s_{n_{\alpha}} \rightarrow s_{0}, \alpha \rightarrow \infty$. Если $s_{0} \notin S$, то опять согласно (1.7) $f\left(s_{n_{\alpha}}, x_{0}\right) \rightarrow-\infty$ при $\alpha \rightarrow \infty$, что противоречит (2.7). Поэтому $s_{0} \in S$. Остальные рассуждения те же, что и в теореме 2.1 .

ТеОРема 2.5. Пусть $X$ - линейное пространство, $S$ - выпуклое подмножество рефлексивного банахова пространства $Y$, а функция $f(\cdot, x)$ коэриитивна в точке $x_{0} \in \operatorname{dom} f$ и пн. сверху и вогнута на множестве $S$ при $x \in X$. Тогда формула (1.2) справедлива для любого направления $\psi \in K\left(x_{0} \mid \operatorname{dom} f\right)$.

ДокаЗАТЕЛЬСТво. Функция (2.14), (2.13) пн. сверху на $Y$. Поскольку она вогнута, то эта функция полунепрерьвна сверху и в слабой топологии [4]. Кроме того, ее множество уровня $(2.15)$ при $\gamma=f\left(s_{1}, x_{0}\right)$ непусто, вьпукло и замкнуто. Из условия (1.8) следует, что оно ограничено, и поэтому в рефлексивном банаховом пространстве $Y$ оно слабо компактно. По теореме Вейерштрасса максимум в (1.1) при $x=x_{0}$ достигается, так что множество (1.3) непусто и слабо компактно.

Пусть элементы $s(\lambda) \in S$ удовлетворяют условию (2.6), и пусть $s_{n}:=s(1 / n)$, $n=1,2, \ldots$ В силу (1.8) и (2.7) эта последовательность ограничена. В рефлексивном пространстве $Y$ можно выбрать подпоследовательность $s_{n_{\alpha}}, \alpha=1,2, \ldots$, слабо сходящуюся к точке $s_{0} \in Y$. Тогда по лемме Мазура [4] найдутся выпуклые комбинации

$$
u_{\alpha}:=\sum_{k=\alpha}^{N} \lambda_{k} s_{n_{k}}, \quad \lambda_{k} \geqslant 0, \quad \sum_{k=\alpha}^{N} \lambda_{k}=1,
$$

сходящиеся к точке $s_{0}$ по норме $Y$ (все числа $N$ и $\lambda_{k}$ зависят от $\alpha$, но здесь это несущественно). 
Но из соотношения (2.7) следует, что числовая последовательность $f\left(s_{n}, x_{0}\right)$, $n=1,2, \ldots$, ограничена снизу некоторой константой $M$. Поскольку множество $S$ выпукло, то $u_{\alpha} \in S, \alpha=1,2, \ldots$, и из вогнутости функции $f\left(\cdot, x_{0}\right)$ на этом множестве вытекает, что

$$
f\left(u_{\alpha}, x_{0}\right) \geqslant \sum_{k=\alpha}^{N} \lambda_{k} f\left(s_{n_{k}}, x_{0}\right) \geqslant \sum_{k=\alpha}^{N} \lambda_{k} M=M
$$

при всех $\alpha=1,2, \ldots$, т.е. числовая последовательность $f\left(u_{\alpha}, x_{0}\right)$ тоже ограничена снизу. Если $s_{0} \notin S$, то это приводит к противоречию с условием $(1.7)$, ибо последовательность $u_{\alpha}, \alpha=1,2, \ldots$, лежит во множестве $S \subset Y$ и сходится к точке $s_{0}$ по норме.

Таким образом, $s_{0} \in S$, а тогда из слабой полунепрерьвности сверху функции $f\left(\cdot, x_{0}\right)$ в точке $s_{0}$ и соотношения $(2.7)$ вытекает равенство $(2.8)$, т.е. $s_{0} \in S\left(x_{0}\right)$. Как и в теореме 2.1, мы получим соотношение (2.11), которое противоречит слабой полунепрерывности функции $f\left(\cdot, x_{0}+\lambda_{1} \psi\right)$ в точке $s_{0} \in S$. Теорема доказана.

ЗАмЕЧАнИЕ 2.2. Максимумы в (1.1) и (1.2) достигаются, если $x=x_{0}$ и $\psi \in$ $K\left(x_{0} \mid \operatorname{dom} f\right)$.

Действительно, как мы видели (см. доказательство теорем 2.1-2.5), множество $S\left(x_{0}\right)$ является непустым компактом, на котором функция $f(\cdot, x)$ пн. сверху (в теореме 2.5 в слабой топологии). Если производная $f^{\prime}\left(x_{0} ; \psi\right)$ конечна, то максимизируемые функции не принимают значения $+\infty$ и пн. сверху на компакте $S\left(x_{0}\right)$ (полунепрерывность сверху производных по направлению следует из того, что нижняя грань

$$
f_{s}^{\prime}\left(x_{0} ; \psi\right)=\inf _{\lambda>0} \frac{\left[f_{s}\left(x_{0}+\lambda \psi\right)-f\left(x_{0}\right)\right]}{\lambda}, s \in S\left(x_{0}\right),
$$

семейства полунепрерывных сверху на множестве $S\left(x_{0}\right)$ функций тоже является полунепрерывной сверху функцией [4]).

ЗАмЕчАниЕ 2.3. Если в условиях теорем 2.1-2.5 функция $f(\cdot, x)$ коэрцитивна во всех точках $x \in X$, для которых $f(s, x)<+\infty$ при всех $s \in S$, то

$$
\operatorname{dom} f=\bigcap_{s \in S} \operatorname{dom} f_{s}
$$

Действительно, во всех случаях

$$
\operatorname{dom} f \subset \bigcap_{s \in S} \operatorname{dom} f_{s}
$$

Если $x \in \operatorname{dom} f_{s}, s \in S$, то число $f(x)$ кончено (это было показано в начале доказательства каждой из теорем 2.1-2.5). 


\section{§3. Субдифференциал максимума выпуклых функций}

Далее, в $\S 3,4,6$, мы будем предполагать, что вьполнены условия любой из теорем 2.1-2.5. Для удобства чтения работы приведем условия этих теорем (как условия 2.1-2.5 соответственно).

2.1. Множество $S$ - компактное топологическое пространство.

2.2. Многозначное отображение $S(x): \operatorname{dom} f \rightarrow S$ имеет непустые компактные значения и пн. сверху на любом отрезке в $\operatorname{dom} f$.

2.3. Множество $S$ относительно компактно в $Y$, а функция $f(s, x)$ коэрцитивна по $s$ в точке $x_{0}$ (см условия (1.7) и (1.8)).

2.4. Множество $S$ - подмножество конечномерного пространства $Y=\mathbb{R}^{m}$, а функция $f(s, x)$ коэрцитивна по $s$ в точке $x_{0}$.

2.5. Множество $S$ - выпуклое подмножество рефлексивного банахова пространства $Y$, а функция $f(s, x)$ коэрцитивна по $s$ в точке $x_{0}$ и вогнута на множестве $S$ при всех $x \in X$.

ТЕОРема 3.1. Пусть $X$ - локально выпуклое пространство и выполнено любое из условий 2.1-2.5. Кроме того, пусть функиии $f(s, x)$ пн. сверху по $s \in S$ при всех $x \in X$ и непрерывны по $x$ в некоторой точке $x_{1} \in \operatorname{dom} f$ nри всех $s \in S$. Тогда если $x_{0}$ - алгебраически внутренняя точка множества $\operatorname{dom} f$, то имеет место равенство

$$
\partial f\left(x_{0}\right)=\overline{\mathrm{conv}} \bigcup_{s \in S\left(x_{0}\right)} \partial f_{s}\left(x_{0}\right)
$$

где замыкание берется в слабой* топологии пространства $X^{*}$.

ДоказАТЕЛЬСТво. Так как $x_{0}$ - алгебраически внутренняя точка $\operatorname{dom} f$, то $K\left(x_{0} \mid \operatorname{dom} f\right)=X$. Тогда из $(2.17)$ вытекает, что $K\left(x_{0} \mid \operatorname{dom} f_{s}\right)=X, s \in S$, т.е. точка $x_{0}$ является алгебраически внутренней для множества $\operatorname{dom} f_{s}, s \in S$. Но поскольку каждая из функций $f_{s}(x)$ непрерывна в точке $x_{1} \in X$, то она не принимает значения $-\infty$ и ее эффективное множество имеет непустую внутренность. Тогда, пользуясь первой теоремой отделимости [4], легко показать, что $x_{0} \in \operatorname{int} \operatorname{dom} f_{s}$ при всех $s \in S$. Кроме того, поскольку выпуклая функция непрерывна внутри своего эффективного множества, если она непрерывна хотя бы в одной точке $x_{1}$ [4], то все функции $f_{s}(x)$ непрерывна в точке $x_{0}$, а потому в ней субдифференцируемы: $\partial f_{s}\left(x_{0}\right) \neq \varnothing, s \in S$.

Для доказательства формулы (3.1) воспользуемся аппаратом опорных функций. Напомним, что опорная функция множества $A \subset X^{*}$ определяется равенством

$$
\delta^{*}(x \mid A)=\sup _{x^{*} \in A}\left\langle x^{*}, x\right\rangle .
$$

Поскольку пространство, сопряженное с пространством $X^{*}$, наделенным слабой* топологией, есть $X$, и сопряженная функция к индикаторной функции $\delta\left(x^{*} \mid A\right)$ равна $\delta^{*}(x \mid A)$, то из теоремы Фенхеля-Моро [4] вытекает, что всякое непустое выпуклое слабо* замкнутое множество $A \subset X^{*}$ можно представить в виде [13]

$$
A=\left\{x^{*} \in X^{*} \mid\left\langle x^{*}, x\right\rangle \leqslant \delta^{*}(x \mid A), x \in X\right\} .
$$


Отсюда сразу же следует, что если два непустых выпуклых слабо* замкнутых множества имеют одинаковые опорные функции, то они совпадают. При вычислении опорных функций мы будем использовать равенства [1], [13]

$$
\delta^{*}(x \mid \overline{\operatorname{conv}} A)=\delta^{*}(x \mid \operatorname{conv} A)=\delta^{*}(x \mid A) .
$$

Опорная функция субдифференциала $\partial f\left(x_{0}\right)$ равна замыканию $\mathrm{cl} f^{\prime}\left(x_{0} ; x\right)$ производной по направлению $f^{\prime}\left(x_{0} ; x\right)[14],[15]$. А опорная функция правой части $(3.1)$ в силу (3.2) равна верхней грани опорных функций субдифференциалов $\partial f_{s}\left(x_{0}\right)$, $s \in S\left(x_{0}\right)$. Поэтому формула (3.1) эквивалентна равенству

$$
\operatorname{cl} f^{\prime}\left(x_{0} ; x\right)=\sup _{s \in S\left(x_{0}\right)} \operatorname{cl} f_{s}^{\prime}\left(x_{0} ; x\right), \quad x \in X,
$$

ибо обе ее части являются непустыми выпуклыми слабо* замкнутыми подмножествами в $X^{*}$ (слабая* замкнутость субдифференциала $\partial f\left(x_{0}\right)$ вытекает непосредственно из его определения, а его непустота - из включения [14]

$$
\bigcup_{s \in S\left(x_{0}\right)} \partial f_{s}\left(x_{0}\right) \subset \partial f\left(x_{0}\right) .
$$

Согласно теоремам 2.1-2.5 для любого направления $\psi \in X=K\left(x_{0} \mid \operatorname{dom} f\right)$ справедлива формула (1.2). Геометрически это означает, что надграфик производной по направлению $f^{\prime}\left(x_{0} ; \cdot\right)$ равен пересечению надпрафиков производных $f_{s}^{\prime}\left(x_{0} ; \cdot\right)$, $s \in S\left(x_{0}\right)$. Поскольку по определению надграфик замыкания функции равен замыканию ее надграфика, то для доказательства равенства (3.3) необходимо проверить, что замыкание пересечения надграфиков производных по направлению $f_{s}^{\prime}\left(x_{0} ; \cdot\right), s \in S\left(x_{0}\right)$, равно пересечению их замыканий.

Как мы видели, все функции $f_{s}(x)$ непрерывны в точке $x_{0}$. Поэтому их производные $f_{s}^{\prime}\left(x_{0} ; \cdot\right)$, непрерывны на всем пространстве $X[4]$. Следовательно, точка

$$
\left(f^{\prime}\left(x_{0} ; \psi_{1}\right)+1, \psi_{1}\right), \quad \psi_{1}:=x_{1}-x_{0},
$$

является внутренней для надграфика каждой из функций $f_{s}^{\prime}\left(x_{0} ; \cdot\right), s \in S\left(x_{0}\right)$ (см. неравенство (2.2)). Поэтому по теореме 6.5 из [15] замыкание пересечения этих надграфиков совпадает с пересечением их замыканий, что доказывает равенство (3.3), а вместе с ним и формулу (3.1). Теорема доказана.

Этот результат обобшает известную теорему о субдифференциале максимума выпуклых функций, полученную в [4] для компактного множества индексов.

ЗАмечаниЕ 3.1 . Как мы видели, все функции $f_{s}(x), s \in S$, непрерывны в точке $x_{0}$, если она является алгебраически внутренней для $\operatorname{dom} f$. В условиях теоремы 2.1 верно и обратное (см. (2.12)).

ЗАмечАниЕ 3.2. Для граничных точек $x_{0}$ формула (3.1) не верна даже для функций одной переменной (например, если $f_{1}(x)=x+1, f_{2}(x)=\delta(x \mid[-\infty, 0])$ и $\left.x_{0}=0\right)$. 
ТЕОРема 3.2. Пусть $X$ - локально выпуклое пространство и выполнено любое из условий 2.1-2.5. Кроме того, пусть функции $f(s, x)$ пн. сверху nо $s \in S$ при всех $x \in X$ и непрерывны по $x$ при всех $s \in S$ в некоторой точке $x_{1} \in \operatorname{dom} f$, которая лежит внутри $\operatorname{dom} f$, если множество $S$ не компактно. Тогда при $x_{0} \in \operatorname{dom} f$

$$
\partial f\left(x_{0}\right)=\overline{\operatorname{conv}} \bigcup_{s \in S\left(x_{0}\right)} \partial f_{s}\left(x_{0}\right)+N\left(x_{0} \mid \operatorname{dom} f\right) .
$$

ДокАЗАТЕЛЬСтво. По лемме 8.2 (см. ниже) субдифференциал $\partial f\left(x_{0}\right)$ будет пуст, только если пусты все субдифференциалы $\partial f_{s}\left(x_{0}\right), s \in S\left(x_{0}\right)$. В этом случае формула (3.6) тривиальна. Пусть теперь множество

$$
S_{0}:=\left\{s \in S\left(x_{0}\right): \partial f_{s}\left(x_{0}\right) \neq \varnothing\right\}
$$

непусто. Тогда в силу (3.4) субдифференциал $\partial f\left(x_{0}\right)$ тоже будет непуст. Поскольку в обеих частях формулы (3.6) стоят непустые (нормальньй конус $N\left(x_{0} \mid \operatorname{dom} f\right.$ ) содержит нулевой вектор) вьпуклые слабо* замкнутые множества в $X^{*}$, то для ее доказательства необходимо проверить, что их опорные функции совпадают.

Сначала заметим, что конус допустимых направлений $K\left(x_{0} \mid \operatorname{dom} f\right)$ является вьпуклым множеством, поэтому индикаторная функция его замыкания выпукла и полунепрерывна снизу. Тогда по теореме Фенхеля-Моро с учетом (3.2) и (1.4) получаем

$$
\begin{aligned}
\delta\left(x \mid \bar{K}\left(x_{0} \mid \operatorname{dom} f\right)\right) & =\delta^{* *}\left(x \mid \bar{K}\left(x_{0} \mid \operatorname{dom} f\right)\right) \\
& =\left(\delta^{*}\right)^{*}\left(x \mid K\left(x_{0} \mid \operatorname{dom} f\right)\right)=\delta^{*}\left(x \mid N\left(x_{0} \mid \operatorname{dom} f\right)\right) .
\end{aligned}
$$

Так как опорная функция суммы множеств равна сумме их опорных функций, то из (3.2) и последнего равенства получаем, что формула (3.6) эквивалентна равенству

$$
\operatorname{cl} f^{\prime}\left(x_{0} ; x\right)=\sup _{s \in S_{0}} \operatorname{cl} f_{s}^{\prime}\left(x_{0} ; x\right)+\delta\left(x \mid \bar{K}\left(x_{0} \mid \operatorname{dom} f\right)\right), \quad x \in X .
$$

Согласно теоремам 2.1-2.5 для любого направления $\psi \in K\left(x_{0} \mid \operatorname{dom} f\right)$ справедлива формула (1.2). Кроме того, очевидно, что $f^{\prime}\left(x_{0} ; \psi\right)=+\infty$ при $\psi \notin$ $K\left(x_{0} \mid \operatorname{dom} f\right)$. Геометрически это означает, что надграфик производной по направлению $f^{\prime}\left(x_{0} ; \cdot\right)$ равен пересечению надграфиков производных $f_{s}^{\prime}\left(x_{0} ; \cdot\right), s \in$ $S\left(x_{0}\right)$, и множества

$$
Z:=\mathbb{R}^{1} \times K\left(x_{0} \mid \operatorname{dom} f\right) .
$$

Таким образом, для доказательства равенства (3.7) необходимо проверить, что замыкание пересечения всех этих множеств равно пересечению замыканий надграфиков производных по направлению $f_{s}^{\prime}\left(x_{0} ; \cdot\right), s \in S_{0}$, и замыкания множества (3.8), равного

$$
\bar{Z}=\mathbb{R}^{1} \times \bar{K}\left(x_{0} \mid \operatorname{dom} f\right) .
$$


Поскольку производные по направлению $f_{s}^{\prime}\left(x_{0} ; \cdot\right)$ непрерывны при $s \in S_{0}$ в точке $\psi_{1}:=x_{1}-x_{0}$ (см. [4, с. 206]), то точка (3.5) является внутренней для надграфика каждой из производных $f_{s}^{\prime}\left(x_{0} ; \cdot\right), s \in S_{0}$. Так как по лемме $8.2 f_{s}^{\prime}\left(x_{0} ; \psi_{1}\right)=-\infty$ при $s \in S\left(x_{0}\right) \backslash S_{0}$, и все функции $f_{s}(x)$ непрерывны в точке $x_{1}$, то (3.5) является внутренней точкой также и для надграфиков производных $f_{s}^{\prime}\left(x_{0} ; \cdot\right), s \in S\left(x_{0}\right) \backslash S_{0}$.

Сначала рассмотрим тот случай, когда $x_{1} \in \operatorname{int} \operatorname{dom} f$. Тогда (3.5)-внутренняя точка множества (3.8), и по теореме 6.5 из [15] мы вновь получим, что замыкание пересечения надграфиков производных по направлению $f_{s}^{\prime}\left(x_{0} ; \cdot\right), s \in S\left(x_{0}\right)$, и множества (3.8) совпадает с пересечением их замыканий, но в этот раз замыкания надграфиков производных по направлению $f_{s}^{\prime}\left(x_{0} ; \cdot\right), s \in S\left(x_{0}\right) \backslash S_{0}$, уже соответствуют несобственным функциям.

Так как эффективное множество производной по направлению $f_{s}^{\prime}\left(x_{0} ; \cdot\right)$ есть $K\left(x_{0} \mid \operatorname{dom} f_{s}\right)$, то из п. в) леммы 8.2 вытекает, что замыкание надграфика такой производной равно

$$
\operatorname{clepi} f_{s}^{\prime}\left(x_{0} ; \cdot\right)=\mathbb{R}^{1} \times \bar{K}\left(x_{0} \mid \operatorname{dom} f_{s}\right), \quad s \in S\left(x_{0}\right) \backslash S_{0} .
$$

Понятно, что пересечение с таким множеством не меняет значения функции, но меняет ее эффективное множество. Однако из соотношения (2.17) вытекает включение

$$
K\left(x_{0} \mid \operatorname{dom} f\right) \subset K\left(x_{0} \mid \operatorname{dom} f_{s}\right), \quad s \in S .
$$

В силу включения (3.11) пересечение множеств (3.10) и (3.9) равно множеству (3.9). Таким образом, замыкание надграфика производной по направлению $f^{\prime}\left(x_{0} ; \cdot\right)$ равно пересечению замыканий надграфиков производных $f_{s}^{\prime}\left(x_{0} ; \cdot\right), s \in S_{0}$, и множества (3.9), что доказывает соотношение (3.7), а вместе с ним и формулу (3.6).

Остается рассмотреть случай, когда $x_{1} \notin \operatorname{int} \operatorname{dom} f$, но множество индексов компактно. В этом случае мы воспользуемся соотношением (2.12), согласно которому можно заменить множество (3.8) на пересечение множеств

$$
\mathbb{R}^{1} \times K\left(x_{0} \mid \operatorname{dom} f_{s}\right), \quad s \in S .
$$

Как мы видели, тогда надграфик производной по направлению $f^{\prime}\left(x_{0} ; \cdot\right)$ равен пересечению надграфииков производных $f_{s}^{\prime}\left(x_{0} ; \cdot\right), s \in S\left(x_{0}\right)$, и множеств (3.12). Поскольку точка (3.5) является внутренней для всех этих множеств, то замыкание их пересечения совпадает с пересечением их замыканий. Замыкания множеств (3.12) равны

$$
\mathbb{R}^{1} \times \bar{K}\left(x_{0} \mid \operatorname{dom} f_{s}\right), \quad s \in S,
$$

поэтому с учетом (3.10) пересечение замыканий надграфиков производных по направлению $f_{s}^{\prime}\left(x_{0} ; \cdot\right), s \in S\left(x_{0}\right)$, и замыканий множеств (3.12) равно пересечению замыканий надграфиков производных $f_{s}^{\prime}\left(x_{0} ; \cdot\right), s \in S_{0}$, и множеств (3.13). Но точка (3.5) является внутренней для каждого из множеств (3.12), поэтому замыкание их пересечения совпадает с пересечением их замыканий, т.е.

$$
\bar{Z}=\bigcap_{s \in S} \mathbb{R}^{1} \times \bar{K}\left(x_{0} \mid \operatorname{dom} f_{s}\right)
$$


Таким образом, замыкание надграфикика производной по направлению $f^{\prime}\left(x_{0} ; \cdot\right)$ равно пересечению замыканий надграфиков производных $f_{s}^{\prime}\left(x_{0} ; \cdot\right), s \in S_{0}$, и множества (3.9), что доказывает соотношения (3.6) и (3.7) для компактного множества индексов. Теорема доказана.

ЗАмЕчАниЕ 3.3. Для некомпактного множества индексов условие $x_{1} \in$ $\operatorname{int} \operatorname{dom} f$ в теоремах 3.1, 3.2 можно заменить на условие (2.12). Для конечного множества индексов $S$ окончательньй результат получил М. Вой [28].

СЛЕДСТвИЕ 3.1. Если в условиях любой из теорем 2.1-2.5 функиия $f(x)$ непрерывна в некоторой точке локально выпуклого пространства $X$, то в точке $x_{0} \in \operatorname{dom} f$ справедлива формула (3.6).

ДокАЗАТЕльСтво. Если выпуклая функция $f(x)$ непрерывна в точке $x_{1} \in X$, то она ограничена сверху в ее окрестности. Тогда будут ограничены сверху и функции $f_{s}(x), s \in S$, и поэтому они тоже непрерывны в этой точке. Остается сослаться на теорему 3.2 .

Формула (3.6) содержит операцию слабого* замыкания и поэтому недостаточно удобна для применения. Дальнейшие наши усилия будут направлены на то, чтобы снять операции замыкания и/или выпуклой оболочки из соотношений (3.1) и (3.6). В частности, как будет видно из следствия 4.2 , в условиях теоремы 2.5 можно снять из (3.1) и (3.6) операции слабого* замыкания и выпуклой оболочки.

\section{§4. Интегральные формулы для субдифференциала}

Определим функции

$$
\begin{aligned}
\tilde{f}_{s}(x): & =f_{s}(x)+\delta(x \mid \operatorname{dom} f)= \begin{cases}f_{s}(x), & x \in \operatorname{dom} f, \\
+\infty & \text { в других случаях. }\end{cases} \\
\tilde{f}(x) & =\sup _{s \in S\left(x_{0}\right)} \tilde{f}_{s}(x) .
\end{aligned}
$$

Легко сообразить, что все они имеют одно и то же эффективное множество:

$$
\operatorname{dom} \tilde{f}_{s}=\operatorname{dom} \tilde{f}=\operatorname{dom} f, \quad s \in S,
$$

и что

$$
f(x) \geqslant \tilde{f}(x), \quad x \in X, \quad f\left(x_{0}\right)=\tilde{f}\left(x_{0}\right) .
$$

Обозначим через $W\left(x_{0}\right)$ множество всех вероятностных мер Радона $\mu$ на компакте $S\left(x_{0}\right)$ (см. замечание 2.2 ).

Теорема 4.1. В условиях теоремы 3.2 субдифференциал функиии $f(x)$ в точке $x_{0} \in \operatorname{dom} f$ можно представить в виде

$$
\begin{aligned}
& \partial f\left(x_{0}\right)=\partial \tilde{f}\left(x_{0}\right)=\overline{\mathrm{conv}} \bigcup_{s \in S\left(x_{0}\right)} \partial \tilde{f}_{s}\left(x_{0}\right), \\
& \partial f\left(x_{0}\right)=\bigcup_{\mu \in W\left(x_{0}\right)} \partial\left(\int \tilde{f}_{s} \mu(d s)\right)\left(x_{0}\right) .
\end{aligned}
$$


ДоКАЗАТЕЛЬСТво. Согласно теореме 3.2 в точке $x_{0}$ справедлива формула (3.6). Сначала заметим, что в силу леммы 8.4 ее можно записать в виде

$$
\partial f\left(x_{0}\right)=\overline{\operatorname{conv}} \bigcup_{s \in S\left(x_{0}\right)}\left[\partial f_{s}\left(x_{0}\right)+N\left(x_{0} \mid \operatorname{dom} f\right)\right] .
$$

Теперь заметим, что поскольку по условию $x_{1} \in \operatorname{dom} f$ и функции $f_{s}(x)$ непрерывны в точке $x_{1}$, то по теореме Моро-Рокафеллара [4]

$$
\begin{aligned}
\partial \tilde{f}_{s}\left(x_{0}\right) & =\partial\left(f_{s}+\delta(\cdot \mid \operatorname{dom} f)\right)\left(x_{0}\right)=\partial f_{s}\left(x_{0}\right)+\partial \delta\left(x_{0} \mid \operatorname{dom} f\right) \\
& =\partial f_{s}\left(x_{0}\right)+N\left(x_{0} \mid \operatorname{dom} f\right),
\end{aligned}
$$

так что из (4.4) и (4.5) мы получаем

$$
\partial f\left(x_{0}\right)=\overline{\mathrm{conv}} \bigcup_{s \in S\left(x_{0}\right)} \partial \tilde{f}_{s}\left(x_{0}\right) .
$$

Далее, из (4.1) следует, что $\partial \tilde{f}\left(x_{0}\right) \subset \partial f\left(x_{0}\right)$, а из (3.4) и слабой* замкнутости субдифференциала следует, что всегда

$$
\overline{\operatorname{conv}} \bigcup_{s \in S\left(x_{0}\right)} \partial \tilde{f}_{s}\left(x_{0}\right) \subset \partial \tilde{f}\left(x_{0}\right) .
$$

Последние два включения и равенство (4.6) дают формулу (4.2). Формула (4.3) следует из теоремы 1.5 из [10] (примененной к функциям $\left.\tilde{f}_{s}(x)\right)$ и равенства $(4.2)$. Теорема доказана.

СлЕДСТВИЕ 4.1. Пусть функиии $f_{s}(x)$ вогнуты по s на выпуклом множестве $S\left(x_{0}\right)$ при всех $x \in \operatorname{dom} f$ и удовлетворяют условиям теоремы 3.2. Тогда при $x_{0} \in \operatorname{dom} f$ имеет место равенство

$$
\partial f\left(x_{0}\right)=\bigcup_{s \in S\left(x_{0}\right)} \partial f_{s}\left(x_{0}\right)+N\left(x_{0} \mid \operatorname{dom} f\right) .
$$

ДокАЗАТЕльСТво. Функции $\tilde{f}_{s}(x)$ пн. сверху и вогнуты по $s$ на выпуклом множестве $S\left(x_{0}\right)$ при всех $x \in X$. Поэтому по леммам 8.5 и 8.6 множество

$$
\bigcup_{s \in S\left(x_{0}\right)} \partial \tilde{f}_{s}\left(x_{0}\right)
$$

будет выпуклым и слабо* замкнутым. Следовательно, можно снять из (4.6) операции слабого* замыкания и выпуклой оболочки, что с учетом (4.5) дает формулу (4.7).

Следуя [10], скажем, что субдифференциал $\partial f\left(x_{0}\right)$ допускает регулярное интегральное представление, если

$$
\partial f\left(x_{0}\right)=\bigcup_{\mu \in W\left(x_{0}\right)} \int \partial f_{s}\left(x_{0}\right) \mu(d s),
$$

т.е. всякий функционал $x^{*} \in \partial f\left(x_{0}\right)$ может быть представлен в виде

$$
\left\langle x, x^{*}\right\rangle=\int\left\langle x, x^{*}(s)\right\rangle \mu(d s) \quad \forall x \in X,
$$

где $\mu \in W\left(x_{0}\right)$ - вероятностная мера Радона на компакте $S\left(x_{0}\right)$, функция $x^{*}(\cdot)$ : $S\left(x_{0}\right) \rightarrow X^{*}$ слабо* $\mu$-суммируема и $x^{*}(s) \in \partial f_{s}\left(x_{0}\right) \mu$-почти всюду на $S\left(x_{0}\right)$. 
СЛЕДСТВИЕ 4.2. Пусть локально выпуклое пространство $X$ сепарабельно, функции $f(s, x)$ пн. сверху по $s \in S$ при всех $x \in X$, а функиия $f(x)$ непрерывна в точке $x_{0} \in X$. Тогда если выполнено любое из условий 2.1-2.5, то субдифференциал $\partial f\left(x_{0}\right)$ допускает регулярное интегральное представление (4.8).

ДоКАЗАТЕЛЬСТВО. По условию точка $x_{0}=x_{1}$ лежит в $\operatorname{int} \operatorname{dom} f$, и выпуклая функция $f(x)$ ограничена в некоторой ее окрестности. Следовательно, выпуклые функции $f_{s}(x), s \in S$, тоже ограничены в этой окрестности и поэтому непрерывны в точке $x_{0}$. Таким образом, условия теоремы 4.1 выполнены, и мы можем воспользоваться представлением (4.3).

Из (4.1) видно, что $x_{0} \in \operatorname{int} \operatorname{dom} \tilde{f}$ и функция $\tilde{f}(x)$ непрерывна в точке $x_{0}$. Тогда по теореме 1.6 из [10], примененной к функциям $\tilde{f}_{s}(x)$, получаем

$$
\partial \tilde{f}\left(x_{0}\right)=\bigcup_{\mu \in W\left(x_{0}\right)} \int \partial \tilde{f}_{s}\left(x_{0}\right) \mu(d s)
$$

Поскольку $x_{0} \in \operatorname{int} \operatorname{dom} f$, то ясно, что $N\left(x_{0} \mid \operatorname{dom} f\right)=\{0\}$. Тогда с учетом (4.5) можно заменить в (4.9) субдифференциалы функций $\tilde{f}_{s}(x)$ на субдифференциалы функций $f_{s}(x)$. Ввиду первого из равенств (4.2) это приводит к интегральному представлению (4.8).

Далее мы покажем, что в случае конечномерного пространства $X=\mathbb{R}^{n}$ можно заменить в (4.8) множество всех вероятностных мер Радона на множество всех дискретных вероятностных мер. В отличие от [9], [10] для этого мы воспользуемся не теоремой Хелли, а излагаемыми ниже результатами.

\section{§5. Замыкания неограниченных выпуклых множеств в конечномерном пространстве}

ПримеР 1. Пусть $X=\mathbb{R}^{2}, f_{1}(x)=0$ при $x_{1}=0$, иначе $f_{1}(x)=+\infty$, а $f_{2}(x) \equiv x_{2}$. Тогда $\partial f_{1}(0)$ - это прямая $x_{2}=0, \partial f_{2}(0)$ - точка $(0,1)$, а их выпуклая оболочка - незамкнутая полоса $0 \leqslant x_{2}<1$ с присоединенной точкой $(0,1)$. Если добавить (алгебраически) к ней нормальный конус $N(0 \mid \operatorname{dom} f)$ - прямую $x_{2}=0,-$ то мы получим замкнутую полосу $0 \leqslant x_{2} \leqslant 1$ - субдифференциал $\partial f(0),-$ так что операция замыкания в формуле (3.6) оказывается лишней.

Следуюшие результаты показывают, что в конечномерном случае $X=\mathbb{R}^{n}$ это не случайно.

Напомним, что если выпуклое множество $C$ содержит некоторьй луч $\{x+\lambda y \mid$ $\lambda \geqslant 0\}$, то направление $y$ называется рецессивным для множества $C$. Назовем $p e$ цессивным конусом $\mathrm{O}^{+} C$ множества $C$ множество всех его рецессивных направлений. В случае замкнутого выпуклого множества $C \subset \mathbb{R}^{n}$ это множество будет целиком содержать все лучи, исходящие из точек $x \in C$, если оно содержит в себе хотя бы один такой луч [15]. Отсюда можно вывести, что $\mathrm{O}^{+} C=\mathrm{O}^{+} \bar{C}$ для любого выпуклого множества $C \subset \mathbb{R}^{n}$. 
ТЕорема 5.1. Если выпуклая оболочка $\operatorname{conv} A$ произвольного множества $A \subset \mathbb{R}^{n}$ не содержит прямых, то

$$
\overline{\operatorname{conv}} A=\operatorname{conv} \bar{A}+\mathrm{O}^{+} \operatorname{conv} A .
$$

По-видимому, впервые этот результат обнаружен Макфадденом [22]. Ввиду его принципиальной важности мы дадим здесь независимое доказательство (см. также [23]).

ДокаЗАТЕЛЬСТво. Пусть $C:=\overline{\operatorname{conv}} A$. Покажем сначала, что

$$
C=\operatorname{conv}\left(\bar{A}+\mathrm{O}^{+} C\right) .
$$

По теореме Кли (см. [15, теорема 10.5]) замкнутое выпуклое множество $C$ является выпуклой оболочкой множества всех своих крайних точек и крайних направлений. Заметим, что любое крайнее направление $y_{0}$ множества $C$ является также крайним направлением рецессивного конуса $\mathrm{O}^{+} C$. Действительно, иначе луч $R:=\left\{x+\lambda y_{0}\right.$, $\lambda \geqslant 0\} \subset C$ является крайним в $C$, но $y_{0}=\lambda_{1} y_{1}+\lambda_{2} y_{2}$, где $y_{i} \in \mathrm{O}^{+} C, \lambda_{i}>0$, $i=1,2$, и $\lambda_{1}+\lambda_{2}=1$, причем векторы $y_{1}$ и $y_{2}$ не коллинеарны $y_{0}$. Тогда в силу замкнутости множества $C$ векторы $x+y_{i}$, не лежашие на луче $R$, будут лежать в $C$ и в то же время $x+y_{0}=\lambda_{1}\left(x+y_{1}\right)+\lambda_{2}\left(x+y_{2}\right)$, т.е. луч $R$ не крайний для множества $C$.

Поэтому достаточно показать, что любая крайняя точка неограниченного выпуклого множества $C$ принадлежит замыканию множества $A$. Сначала пусть $\bar{x}-$ выступаюшая точка $C$. Тогда $\bar{x}=\nabla \delta^{*}(\psi \mid C)=\nabla \delta^{*}(\psi \mid \bar{A})$ в некоторой точке $\psi$. Поскольку $\delta^{*}(\psi \mid \bar{A})$ - сопряженная функция к пн. снизу функции $\delta(\psi \mid \bar{A})$, то по теореме 1 из [17] (см. также [25]) получаем, что $\delta(\bar{x} \mid \bar{A})=\delta^{* *}(\bar{x} \mid \bar{A})=\delta(\bar{x} \mid C)=0$, т.е. $\bar{x} \in A$. По теореме Страшевича $[15]$ каждая крайняя точка множества $C$ является пределом его выступаюших точек. Но мы сейчас показали, что все выступаюшие точки множества $C$ лежат в множестве $\bar{A}$. Поэтому в силу замкнутости множества $\bar{A}$ оно содержит и все крайние точки множества $C$.

Теперь равенство (5.1) вытекает из (5.2) в силу леммы 8.4 и соотношения $\mathrm{O}^{+} \mathrm{C}=$ $\mathrm{O}^{+} \operatorname{conv} A$. Теорема доказана.

Формула (5.1) может не иметь места, если выпуклая оболочка множества $A$ содержит прямую, что показывает

ПримеР 2. Пусть замкнутое множество $A \subset \mathbb{R}^{2}$ состоит из точек $(k,(1+$ $\left.|k|)^{-1}\right), \quad k=0, \pm 1, \pm 2, \ldots$ Тогда его выпуклая оболочка conv $A$ - это открытая полоса $0<x_{2}<1$ с присоединенной точкой $(0,1)$. Добавив (алгебраически) к ней рецессивный конус $\mathrm{O}^{+} \operatorname{conv} A-$ прямую $x_{2}=0,-$ получим незамкнутую полосу $0<x_{2} \leqslant 1$.

Однако имеет место

Теорема 5.2. Пусть $A$ - произвольное множество в $\mathbb{R}^{n}$, целиком содержащее любую прямую, пересекающ,ую әто множество, если она параллельна некоторой прямой, принадлезсащей его выпуклой оболочке. Тогда справедливо равенство (5.1). 
ДокАЗАТЕЛЬСТво. Обозначим через $L:=\mathrm{O}^{+} C \cap\left\{-\mathrm{O}^{+} C\right\}$ линейное подмножество множества $C$. Поскольку по условию $A+L \subset A$, то, как легко видеть, замыкание, выпуклую оболочку и рецессивный конус для множества $A$ можно сначала вычислить не для самих этих множеств, а для их пересечений с ортогональным дополнением $L^{\perp}$ подпространства $L$, а затем добавить (алгебраически) $L$. Очевидно, что множество conv $A \cap L^{\perp}$ уже не содержит прямых. Поэтому по теореме 5.1 утверждение верно на подпространстве $L^{\perp}$, а потому и во всем пространстве. Теорема доказана.

СЛЕДСТВИЕ 5.1. Если замкнутое множество $A \subset \mathbb{R}^{n}$ является обгединением семейства выпуклых замкнутых множеств $A_{\gamma}, \gamma \in \Gamma$, таких, что $\mathrm{O}^{+} A_{\gamma}=\mathrm{O}^{+} \operatorname{conv} A$ при всех $\gamma \in \Gamma$, то его выпуклая оболочка замкнута и

$$
\overline{\operatorname{conv}} A=\left\{\sum_{j=1}^{n+1} \alpha_{j} x_{j}: x_{j} \in A_{\gamma_{j}}, \gamma_{j} \in \Gamma, \alpha_{j} \geqslant 0,1 \leqslant j \leqslant n+1, \sum_{j=1}^{n+1} \alpha_{j}=1\right\}
$$

ДокАЗАТЕЛЬство. По условию все вьпуклые множества $A_{\gamma}, \gamma \in \Gamma$, и conv $A$ имеют общее линейное подмножество $L$, поэтому $A+L \subset A$, и по теореме 5.2 любая точка $x \in \overline{\operatorname{conv}} A$ имеет вид $x=x_{0}+y$, где $y \in \mathrm{O}^{+} \operatorname{conv} A$, a $x_{0}=\alpha_{1} x_{1}+\cdots+\alpha_{k} x_{k}$ - выпуклая комбинация некоторых точек $x_{j} \in A_{\gamma_{j}}, \gamma_{j} \in \Gamma, j=1, \ldots, k$. Тогда точка $x=\alpha_{1}\left(x_{1}+y\right)+\cdots+\alpha_{k}\left(x_{k}+y\right)$ является выпуклой комбинацией точек $x_{j}+y \in A_{\gamma_{j}}, j=1, \ldots, k$ (ибо рецессивные конусы множеств $A_{\gamma_{j}}$ совпадают с $\mathrm{O}^{+} \operatorname{conv} A$ и эти множества замкнуты). Это означает, что $x \in \operatorname{conv} A$, т.е. множество conv $A$ замкнуто. Формула (5.3) теперь вытекает из теоремы Каратеодори [4].

СЛЕДСТВИЕ 5.2. Если множество $A \subset \mathbb{R}^{n}$ является обгединением семейства выпуклых множеств $A_{\gamma}, \quad \gamma \in \Gamma$, maких, что $\mathrm{O}^{+} A_{\gamma}=\mathrm{O}^{+} \operatorname{conv} A$ при всех $\gamma \in \Gamma$, то справедливо равенство (5.1).

ДокАЗАТЕльство. Если прямая $\left\{x+\lambda y_{0}, \lambda \in \mathbb{R}\right\}$ принадлежит выпуклой оболочке conv $A$ множества $A$, то вектор $y_{0}$ лежит в линейном подмножестве $L$ множества conv $A$, которое совпадает с линейным подмножеством каждого из выпуклых множеств $A_{\gamma}, \gamma \in \Gamma$. Поэтому $A+L \subset A$, т.е. выполнены условия теоремы 5.2 .

СЛЕДСТВИЕ 5.3. Пусть множсество $A \subset \mathbb{R}^{n}$ является объединением выпукльх замкнутых множеств $A_{s}, s \in S$, имеющих общий рецессивный конус. Кроме того, пусть множество $S$ будет компактным топологическим пространством и опорная функиия $\delta^{*}\left(x \mid A_{s}\right)$ полунепрерывна сверху по $s$ на $S$ при каждом $x \in \mathbb{R}^{n}$. Тогда множества $A$ и conv $A$ будут замкнуты, а рецессивный конус множеств $A_{s}, s \in S$, равен рецессивному конусу множества $\operatorname{conv} A$.

ДоказАтЕльство. Опорная функция $\delta^{*}(x \mid A)$ множества $A$ равна верхней грани опорных функций $\delta^{*}\left(x \mid A_{s}\right)$, взятой по $s \in S$. Поскольку они пн. сверху по $s$ на компакте $S$ при каждом $x \in \mathbb{R}^{n}$, то по теореме Вейерштрасса эта верхняя грань конечна для тех $x$, для которых все числа $\delta^{*}\left(x \mid A_{s}\right), s \in S$, конечны. Иначе говоря, 
с учетом $(3.2)$

$$
\operatorname{dom} \delta^{*}(\cdot \mid \operatorname{conv} A)=\bigcap_{s \in S} \operatorname{dom} \delta^{*}\left(\cdot \mid A_{s}\right)
$$

Теперь воспользуемся тем, что рецессивный конус $\mathrm{O}^{+} A_{s}$ является полярой барьерного конуса $\operatorname{dom} \delta^{*}\left(\cdot \mid A_{s}\right)$ (см. [13, с. 35]). Так как по условию множества $A_{s}$, $s \in S$, имеют обший рецессивный конус, то поляры конусов $\mathrm{O}^{+} A_{s}$, равные замыканиям барьерных конусов $\operatorname{dom} \delta^{*}\left(\cdot \mid A_{s}\right), s \in S$, тоже совпадают. Следовательно, конусы $\operatorname{dom} \delta^{*}\left(\cdot \mid A_{s}\right), s \in S$, имеют общую относительную внутренность. Тогда в силу (5.4) ту же самую относительную внутренность имеет и конус $\operatorname{dom} \delta^{*}(\cdot \mid \operatorname{conv} A)$. Поскольку поляра любого конуса совпадает с полярой его относительной внутренности, то отсюда следует, что рецессивные конусы $\mathrm{O}^{+} A_{s}$, $s \in S$, совпадают с рецессивным конусом $\mathrm{O}^{+} \operatorname{conv} A$.

Докажем замкнутость множества $A$. Пусть точка $a$-предел точек $a_{j} \in A$. Тогда $a_{j} \in A_{s_{j}}$ для некоторых элементов $s_{j} \in S$. В силу компактности множества $S$ можно считать, что $s_{j} \rightarrow s \in S$. Так как многозначное отображение $A_{s}: S \rightarrow \mathbb{R}^{n}$ имеет выпуклые замкнутые значения, а их опорная функция $\delta^{*}\left(x \mid A_{s}\right)$ пн. сверху по $s$ на $S$ при каждом $x \in \mathbb{R}^{n}$, то оно имеет замкнутый график (см. [13, с. 128]). Поэтому $a \in A_{s}$ для некоторого $s \in S$. Тем самым множество $A$ замкнуто.

Для завершения доказательства остается сослаться на следствие 5.3.

Для конечного множества индексов последний результат известен (см. следствие 9.8.1 из [15]).

Возвращаясь к субдифференциалу максимума выпуклых функций, заметим, что согласно лемме $8.3 N\left(x_{0} \mid \operatorname{dom} f\right)=\mathrm{O}^{+} \partial f\left(x_{0}\right)$. С учетом следствия 5.1 это позволяет в конечномерном случае снять операцию замыкания из формул (3.1) и (3.6) (см. далее формулы (7.2) и (6.1) соответственно).

\section{§6. Конечномерный случай}

ТЕОРема 6.1. Пусть $X=\mathbb{R}^{n}$ - конечномерное пространство и выполнено любое из условий 2.1-2.5. Кроме того, пусть функиии $f(s, x)$ пн. сверху по $s \in S$ при всех $x \in X$ и найдется точка $x_{1} \in \operatorname{ridom} f_{s}, s \in S$, лежсащая в ridom $f$, если множество $S$ не компактно. Тогда при $x_{0} \in \operatorname{dom} f$ имеем

$$
\begin{aligned}
\partial f\left(x_{0}\right)=\{ & \left\{\sum_{j=1}^{n+1} \alpha_{j} x_{j}^{*}+x_{0}^{*} \mid x_{j}^{*} \in \partial f_{s_{j}}\left(x_{0}\right), s_{j} \in S\left(x_{0}\right), \alpha_{j} \geqslant 0\right. \\
& \left.j=1, \ldots, n+1, \sum_{j=1}^{n+1} \alpha_{j}=1, x_{0}^{*} \in N\left(x_{0} \mid \operatorname{dom} f\right)\right\} .
\end{aligned}
$$

Более того, в случае условия 2.5 справедлива формула (4.7).

ДокаЗАТЕЛЬСтво. Поскольку $x_{1} \in \operatorname{ridom} f_{s}$, то $\partial f_{s}\left(x_{1}\right) \neq \varnothing[15]$, и поэтому функции $f_{s}(x)$ не принимают значение $-\infty$. Покажем, что $x_{1} \in \operatorname{ridom} f$ и в том случае, когда множество $S$ компактно. Действительно, так как $x_{1} \in \operatorname{ridom} f_{s}$, 
то $K\left(x_{1} \mid \operatorname{dom} f_{s}\right)$ - линейное подпространство при всех $s \in S$. Поэтому согласно (2.12) конус $K\left(x_{1} \mid \operatorname{dom} f\right)$, равньй пересечению этих подпространств, тоже будет линейным подпространством. А это означает, что $x_{1} \in \operatorname{ridom} f$, а потому вектор $\psi_{1}=x_{1}-x_{0}$ является относительно внутренним в конусах допустимых направлений $K\left(x_{0} \mid \operatorname{dom} f\right)$ и $K\left(x_{0} \mid \operatorname{dom} f_{s}\right), s \in S$ (очевидно, что это справедливо и для некомпактного множества $S)$.

Теперь докажем формулу (3.6). Для этого воспользуемся тем, что в конечномерном пространстве субдифференциал выпуклой функции будет пустым только тогда, когда производные по всем относительно внутренним направлениям равны $-\infty[15]$. Поскольку вектор $\psi_{1}$ является относительно внутренним в конусах $K\left(x_{0} \mid \operatorname{dom} f\right)$ и $K\left(x_{0} \mid \operatorname{dom} f_{s}\right)$, то из формулы (1.2) вытекает, что субдифференциал $\partial f\left(x_{0}\right)$ будет пуст лишь в том случае, когда будут пусты все субдифференциалы $\partial f_{s}\left(x_{0}\right), s \in S\left(x_{0}\right)$. В этом случае формула (3.6), очевидно, выполняется.

Пусть теперь субдифференциалы $\partial f_{s}\left(x_{0}\right)$ не пусты при $s \in S_{0}$. Тогда в обеих частях формулы (3.6) будут стоять непустые выпуклые замкнутые множества, причем опорная функция правой части имеет вид (3.7). Кроме того, по теоремам 2.1-2.5 надграфик производной по направлению $f^{\prime}\left(x_{0} ; \cdot\right)$ равен пересечению надграбиков производных $f_{s}^{\prime}\left(x_{0} ; \cdot\right), s \in S\left(x_{0}\right)$, и множества $(3.8)$.

Так как эффективным множеством производной $f_{s}^{\prime}\left(x_{0} ; \cdot\right)$ является конус допустимых направлений $K\left(x_{0} \mid \operatorname{dom} f_{s}\right)$, то вектор $\psi_{1}$ является относительно внутренним для этих эффективных множеств. Поскольку он является относительно внутренним и в конусе $K\left(x_{0} \mid \operatorname{dom} f\right)$, то $f^{\prime}\left(x_{0} ; \psi_{1}\right) \neq+\infty$. А так как $\partial f\left(x_{0}\right) \neq \varnothing$, то $f^{\prime}\left(x_{0} ; \psi_{1}\right) \neq-\infty$, т.е. эта производная конечна. Тогда в силу неравенства $(2.2)$ точка (3.5) будет относительно внутренней для надграфика каждой из производных $f_{s}^{\prime}\left(x_{0} ; \cdot\right), s \in S\left(x_{0}\right)$. Очевидно, что она относительно внутренняя и для множества (3.8). По теореме 6.5 из [15] замыкание пересечения всех этих множеств равно пересечению их замыканий.

Поскольку $f_{s}^{\prime}\left(x_{0} ; \psi\right)=-\infty$ при $s \in S\left(x_{0}\right) \backslash S_{0}$ для всех относительно внутренних направлений конуса $K\left(x_{0} \mid \operatorname{dom} f_{s}\right)$, то замыкания надграфиков производных $f_{s}^{\prime}\left(x_{0} ; \cdot\right)$ при $s \in S\left(x_{0}\right) \backslash S_{0}$ дают множества (3.10). А замыкание множества (3.8) имеет вид (3.9). В силу включения (3.11) пересечение множеств (3.10) и (3.9) равно множеству (3.9). Таким образом, замыкание надграфика производной по направлению $f^{\prime}\left(x_{0} ; \cdot\right)$ равно пересечению замыканий надпрафиков производных $f_{s}^{\prime}\left(x_{0} ; \cdot\right), s \in S_{0}$, и множества (3.9). Как мы видели, это доказывает формулу (3.6).

Теперь заметим, что справедливо также равенство (4.2). Действительно, его доказательство, данное в теореме 4.1, опиралось лишь на равенства (3.6) и (4.5). Первое из них, как мы только что показали, выполняется. А второе следует из теоремы 23.8 из [15], поскольку во всех случаях $x_{1} \in \operatorname{ridom} f \cap \operatorname{ridom} f_{s}, s \in S$.

Далее, по лемме 8.3 в силу (4.0) все субдифференциалы $\partial \tilde{f}_{s}\left(x_{0}\right), s \in S\left(x_{0}\right)$, и $\partial \tilde{f}\left(x_{0}\right)$ имеют один и тот же рецессивный конус. Они выпуклы и замкнуты, а по лемме 8.6 будет замкнутым и объединение всех субдифференциалов $\partial \tilde{f}_{s}\left(x_{0}\right)$, $s \in S\left(x_{0}\right)$. Тогда по следствию 5.1 выпуклая оболочка этого объединения тоже замкнута. Это означает, что из формулы (4.2) можно убрать замыкание:

$$
\partial f\left(x_{0}\right)=\operatorname{conv} \bigcup_{s \in S\left(x_{0}\right)} \partial \tilde{f}_{s}\left(x_{0}\right) .
$$


Используя теорему Каратеодори [4], отсюда получаем, что

$$
\begin{array}{r}
\partial f\left(x_{0}\right)=\left\{\sum_{j=1}^{n+1} \alpha_{j} \tilde{x}_{j}^{*}: \tilde{x}_{j}^{*} \in \partial \tilde{f}_{s_{j}}\left(x_{0}\right), s_{j} \in S\left(x_{0}\right),\right. \\
\left.\alpha_{j} \geqslant 0,1 \leqslant j \leqslant n+1, \sum_{j=1}^{n+1} \alpha_{j}=1\right\} .
\end{array}
$$

Формула (6.1) теперь следует из доказанного нами равенства (4.5) и выпуклости нормального конуса $N\left(x_{0} \mid \operatorname{dom} f\right)$. В условиях теоремы 2.5 по лемме 8.5 из формулы (4.2) можно убрать не только операцию замыкания, но и выпуклой оболочки, что дает формулу (4.7). Теорема доказана.

Любопытно, что в том случае, когда все функции $f_{s}(x), s \in S$, имеют общую область определения $\operatorname{dom} f_{s}=\operatorname{dom} f:=M, s \in S$, нормальный конус $N\left(x_{0} \mid \operatorname{dom} f\right)$ "прячется" в субдифференциалах $\partial f_{s}\left(x_{0}\right), s \in S$. В такой форме (при дополнительных условиях замкнутости множества $M$ и полунепрерывности снизу функций $f_{s}(x), s \in S$, на этом множестве) формула (6.1) получена В. Л. Левиным (см. [9], [10]). Очевидно, что нормальный конус исчезает из формулы (6.1) и в том случае, когда точка $x_{0}$ лежит внутри множества $\operatorname{dom} f$.

\section{§ 7. Случай произвольного множества индексов}

Далее $S$ - произвольное множество индексов. В теоремах 7.1, 7.3 предполагается, что в (1.1) достигается максимум. Обозначим через $\operatorname{dom}\left(\partial f^{*}\right)$ множество всех векторов $x^{*} \in X^{*}$ таких, что $\partial f^{*}\left(x^{*}\right) \neq \varnothing$.

В тех случаях, когда явно не оговаривается противное, $X$ будет локально выпуклым пространством.

Теорема 7.1. Пусть $f_{s}(x): X \rightarrow \overline{\mathbb{R}}, s \in S,-$ произвольное семейство пн. снизу выпуклых функций, и пусть множество $S(x)$ не пусто при всех $x \in \operatorname{dom}(\partial f)$. Тогда следующие свойства әквивалентны:

a) $\partial f(x)=\bigcup_{s \in S(x)} \partial f_{s}(x)$ nри всех $x \in \operatorname{dom} f$;

б) множество $S^{*}\left(x^{*}\right):=\left\{s \in S \mid f_{s}^{*}\left(x^{*}\right)=f^{*}\left(x^{*}\right)\right\}$ не пусто при всех $x^{*} \in \operatorname{dom}\left(\partial f^{*}\right)$

в) множество $S(x) \cap S^{*}\left(x^{*}\right)$ не пусто для всех $x^{*} \in \partial f(x)$;

г) если $x^{*} \in \partial f(x)$, то $x^{*} \in \partial f_{s}(x)$ для некоторого $s \in S(x) \cap S^{*}\left(x^{*}\right)$;

д) если $x \in \partial f^{*}\left(x^{*}\right)$, то $x \in \partial f_{s}^{*}\left(x^{*}\right) \partial л я$ некоторого $s \in S(x) \cap S^{*}\left(x^{*}\right)$.

В этом случае

$$
f^{*}\left(x^{*}\right)=\min _{s \in S} f_{s}^{*}\left(x^{*}\right), \quad x^{*} \in \operatorname{dom}\left(\partial f^{*}\right)
$$

ДокАЗАТЕльство. Эквивалентность этих свойств докажем по схеме а) $\Leftrightarrow$ б) $\Rightarrow$ д) $\Leftrightarrow г) \Rightarrow$ в $\Rightarrow$ б). Если $x^{*} \in \operatorname{dom}\left(\partial f^{*}\right)$, то найдется вектор $x \in \partial f^{*}\left(x^{*}\right)$. В силу полунепрерывности снизу функции $f(x)$ поэтому $x^{*} \in \partial f(x)$, т.е. $x \in \operatorname{dom}(\partial f)$. Тогда по лемме 8.8 из а) следует б). 
Наоборот, пусть выполнено б) и $x \in \operatorname{dom}(\partial f)$ (иначе свойство а) тривиально). Тогда найдется $x^{*} \in \partial f(x)$. Следовательно, $x \in \partial f^{*}\left(x^{*}\right)$, т.е. $x^{*} \in \operatorname{dom}\left(\partial f^{*}\right)$. По условию б) поэтому $S^{*}\left(x^{*}\right) \neq \varnothing$ и по лемме 8.7 найдется элемент $s \in S(x) \cap S^{*}\left(x^{*}\right)$ такой, что $x \in \partial f_{s}^{*}\left(x^{*}\right)$, т.е. $x^{*} \in \partial f_{s}(x)$ в силу полунепрерывности снизу функции $f_{s}(x)$. В силу произвольности элемента $x^{*} \in \partial f(x)$ это означает, что

$$
\partial f(x) \subset \bigcup_{s \in S(x)} \partial f_{s}(x)
$$

Но обратное включение очевидно: если $x^{*} \in \partial f_{s}(x)$ и $f_{s}(x)=f(x)$, то

$$
f(\tilde{x})-f(x) \geqslant f_{s}(\tilde{x})-f_{s}(x) \geqslant\left\langle x^{*}, \tilde{x}-x\right\rangle \quad \forall \tilde{x} \in X,
$$

т.e. $x^{*} \in \partial f(x)$.

Утверждение д) следует из б) по лемме 8.7. Свойство д) эквивалентно г) в силу полунепрерывности снизу функций $f_{s}(x)$ и $f(x)$. То, что из г) следует в), а из в) следует б), очевидно. Теорема доказана.

В [25] дан содержательный пример применения равенства (7.1).

Теорема 7.2. Пусть $f_{s}(x): \mathbb{R}^{n} \rightarrow \overline{\mathbb{R}}, s \in S$, - произвольное семейство выпукльих функиий, и пусть $\tilde{f}_{s}(x):=f_{s}(x)$, если $x \in \operatorname{dom} f$, а иначе $\tilde{f}_{s}(x):=+\infty$. Предположим, ито нижняя грань $\inf _{s \in S} \tilde{f}_{s}^{*}\left(x^{*}\right)$ достигается всюду, где она конечна и пн. снизу на $\mathbb{R}^{n}$ (как функция переменной $\left.x^{*}\right)$. Тогда при всех $x_{0} \in \operatorname{dom} f$ имеет место равенство

$$
\partial f\left(x_{0}\right)=\left\{\sum_{j=1}^{n+1} \alpha_{j} x_{j}^{*}: x_{j}^{*} \in \partial \tilde{f}_{s_{j}}\left(x_{0}\right), s_{j} \in S\left(x_{0}\right), \alpha_{j} \geqslant 0, \sum_{j=1}^{n+1} \alpha_{j}=1\right\} .
$$

ДокАЗАтЕльство. Объединение выпуклых замкнутых множеств ері $\tilde{f}_{s}^{*}, s \in S$, замкнуто по предположению. Поскольку $\operatorname{dom} \tilde{f}_{s}=\operatorname{dom} f$ для всех $s \in S$, то эти множества имеют обший рецессивньй конус, совпадающий с рецессивным конусом надграфика ері $f^{*}$ (см. [15, теорема 13.3]). Поэтому согласно следствию 5.1 (см. также [15, теорема 16.5]) надграфик ері $f^{*}$ является выпуклой оболочкой этого объединения, т.е. для всех $x^{*} \in \operatorname{dom} f^{*}$ имеем

$$
f^{*}\left(x^{*}\right)=\sum \alpha_{j} \beta_{j}, \quad x^{*}=\sum \alpha_{j} x_{j}^{*}
$$

где

$$
\beta_{j} \geqslant \tilde{f}_{s_{j}}^{*}\left(x_{j}^{*}\right), \quad s_{j} \in S, \quad \alpha_{j}>0, \quad \sum \alpha_{j}=1 .
$$

Теперь пусть $x^{*} \in \partial f(x)$. Тогда [15]

$$
f(x)+f^{*}\left(x^{*}\right)=\left\langle x, x^{*}\right\rangle .
$$


Поскольку $f(x) \geqslant \sum \alpha_{j} f_{s_{j}}(x)$, то из (7.3)-(7.5) получаем, что

$$
\sum \alpha_{j} f_{s_{j}}(x)+\sum \alpha_{j} \tilde{f}_{s_{j}}^{*}\left(x_{j}^{*}\right) \leqslant \sum \alpha_{j}\left\langle x, x_{j}^{*}\right\rangle .
$$

С другой стороны, поскольку $x \in \operatorname{dom} f$, то $\tilde{f}_{s}(x)=f_{s}(x)$, поэтому

$$
f_{s_{j}}(x)+\tilde{f}_{s_{j}}^{*}\left(x_{j}^{*}\right) \geqslant\left\langle x, x_{j}^{*}\right\rangle
$$

для всех индексов $j$. Последние два неравенства могут вьполняться, лишь только когда достигаются равенства

$$
f_{s_{j}}(x)+\tilde{f}_{s_{j}}^{*}\left(x_{j}^{*}\right)=\left\langle x, x_{j}^{*}\right\rangle, \quad f_{s_{j}}(x)=f(x),
$$

что эквивалентно включениям $x_{j}^{*} \in \partial \tilde{f}_{s_{j}}(x)$ и $s_{j} \in S(x)$ для всех индексов $j$. Таким образом, субдифференциал $\partial f(x)$ принадлежит правой части формулы (7.2). Обратное включение тривиально. Теорема доказана.

Условия теоремы 7.2 выполняются в том случае, когда все функции $f_{s}(x)$ пн. сверху по $s$ на компакте $S$ при любом $x \in \operatorname{dom} f$ и имеют общую область определения $\operatorname{dom} f_{s}=\operatorname{dom} f:=M, s \in S$ (см. [9], [10]).

Теорема 7.3. Пусть $f_{s}(x): X \rightarrow \overline{\mathbb{R}}, s \in S,-$ произвольное семейство выпуклых функций таких, что функция $f(x)$ непрерывна в некоторой точке $x_{1} \in X$, и множество $S(x)$ не пусто при всех $x \in \operatorname{int} \operatorname{dom} f$. Тогда следующие утверждения әквивалентны:

а) при всех $x_{0} \in \operatorname{int} \operatorname{dom} f$ справедливо равенство (3.1);

б) верхняя грань $\sup _{s \in S(x)} f_{s}^{\prime}(x ; \psi)$ пн. сверху по $x$ на любом отрезке внутpu $\operatorname{dom} f$ при фиксированных $\psi \in X$.

В этом случае при всех $x_{0} \in \operatorname{int} \operatorname{dom} f$ имеет место равенство (1.2).

Доказательство. Рассмотрим многозначное отображение $F(x): \operatorname{int} \operatorname{dom} f \rightarrow$ $X^{*}$ с непустыми выпуклыми слабо* компактными значениями:

$$
F(x):=\overline{\mathrm{conv}} \bigcup_{s \in S(x)} \partial f_{s}(x)
$$

Действительно, из непрерывности функции $f(x)$ следует непрерывность, а потому и субдифференцируемость функций $f_{s}(x)$ на множествах int $\operatorname{dom} f_{s}(x) \supset$ $\operatorname{int} \operatorname{dom} f(x), s \in S$, поэтому множества $F(x)$ не пусты. А поскольку $F(x) \subset$ $\partial f(x)$ (см. (4.6а)), то $F(x)$ - слабо* замкнутое подмножество слабо* компактного субдифференциала $\partial f(x)$. При $x \in \operatorname{int} \operatorname{dom} f$ его опорная функция равна (см. (3.2))

$$
\delta^{*}(\psi \mid F(x))=\sup _{s \in S(x)} \delta^{*}\left(\psi \mid \partial f_{s}(x)\right)=\sup _{s \in S(x)} f_{s}^{\prime}(x ; \psi) .
$$

По условию она пн. сверху по $x$, поэтому многозначное отображение $\lambda \rightarrow F\left(x_{0}+\right.$ $\lambda \psi$ ) имеет замкнутый график (см. $[13$, с. 128$]$; в дальнейшем пространство $X^{*}$ рассматривается в слабой* топологии). 
Итак, $F(x) \subset \partial f(x)$. Предположим, что найдутся $x_{0} \in \operatorname{int} \operatorname{dom} f$ и $x_{0}^{*} \in \partial f\left(x_{0}\right)$ такие, что $x_{0}^{*} \notin F\left(x_{0}\right)$. Тогда по второй теореме отделимости найдутся вектор $\psi \in X, \psi \neq 0$, и число $\varepsilon>0$ такие, что [4]

$$
\left\langle x^{*}, \psi\right\rangle \leqslant\left\langle x_{0}^{*}, \psi\right\rangle-\varepsilon \forall x^{*} \in F\left(x_{0}\right) .
$$

Рассмотрим направленность $x(\lambda):=x_{0}+\lambda \psi, \delta \geqslant \lambda \geqslant 0$, в которой $\delta$ достаточно мало, так что $x(\lambda) \in \operatorname{int} \operatorname{dom} f$ при всех $\lambda \in[0, \delta]$. Выберем любые элементы $x^{*}(\lambda) \in F(x(\lambda))$. Поскольку субдифференциальное отображение $\lambda \rightarrow \partial f(x(\lambda))$ пн. сверху [13] и имеет слабо* компактные значения (см. [8, с. 176]), то образом компакта $[0, \delta]$ при таком отображении будет слабо* компактноемножество [13], в котором и будут лежать векторы $x^{*}(\lambda) \in F(x(\lambda)) \subset \partial f(x(\lambda))$. Следовательно, из направленности $x^{*}(\lambda), \delta \geqslant \lambda \geqslant 0$, в которой индекс $\lambda$ направлен по убыванию, можно выделить поднаправленность $x^{*}(\lambda(\alpha)), \alpha \in A$, слабо* сходящуюся к элементу $x^{*}$. Так как $\lambda(\alpha) \rightarrow 0$ и многозначное отображение $\lambda \rightarrow F\left(x_{0}+\lambda \psi\right)$ имеет замкнутый график, то $x^{*} \in F\left(x_{0}\right)$. Поскольку субдифференциальное отображение $x \rightarrow \partial f(x)$ монотонно, то $\left\langle x^{*}(\lambda(\alpha))-x_{0}^{*}, x(\lambda(\alpha))-x_{0}\right\rangle \geqslant 0$, т.е. $\left\langle x^{*}(\lambda(\alpha))-x_{0}^{*}, \psi\right\rangle \geqslant 0$. В пределе получим $\left\langle x^{*}-x_{0}^{*}, \psi\right\rangle \geqslant 0$, что противоречит неравенству (7.7).

Таким образом, $x_{0}^{*} \in F\left(x_{0}\right)$, что доказывает (3.1) для всех $x_{0} \in \operatorname{int} \operatorname{dom} f$. Наоборот, пусть выполнено утверждение а). Тогда в силу (7.6) при всех $x_{0} \in$ int $\operatorname{dom} f$ будет справедливо равенство (1.2), и из непрерьвности функции $f(x)$ будет следовать полунепрерывность сверху производной $f^{\prime}\left(x_{0} ; \psi\right)$ (см. [13], [15]), а вместе с ней и правой части (1.2) по переменной $x_{0}$ при каждом $\psi \in X$. Теорема доказана.

Последние две теоремы возникли под влиянием красивых результатов, принадлежащих М. Вою и А. В. Тимохову (см. [28, с. 150] и [20, с. 107] соответственно).

СлЕДСТВИЕ 7.1. Пусть $f_{s}(x): X \rightarrow \overline{\mathbb{R}}, s \in S,-$ произвольное семейство выпуклых функций, дифференцируемых по Гато на $\operatorname{int} \operatorname{dom} f, \operatorname{ma\kappa их,~что~}$ функиия $f(x)$ непрерывна в некоторой точке $x_{1} \in X$, и множество $S(x)$ одноточечно при всех $x \in \operatorname{int} \operatorname{dom} f$. Тогда следующие утверждения әквивалентны:

а) функция $f(x)$ дифферениируема по Гато на int dom $f$;

б) функиия $\left\langle\nabla f_{S(x)}(x), \psi\right\rangle$ непрерывна по $x$ на любом отрезке внутри $\operatorname{dom} f$ при фиксированных $\psi \in X$.

В этом случае $\nabla f(x)=\nabla f_{S(x)}(x)$ при всех $x \in \operatorname{int} \operatorname{dom} f$.

ДокАЗАТЕЛЬСТВО. Если функции $f_{s}(x)$ дифференцируемы по Гато, то $\partial f_{s}(x)=$ $\left\{\nabla f_{s}(x)\right\}[21]$, поэтому формула (3.1) имеет вид $\partial f(x)=\left\{\nabla f_{S(x)}(x)\right\}$. Так как функция $f(x)$ непрерывна на int $\operatorname{dom} f$, то она дифференцируема по Гато [21].

СЛЕДСТВИЕ 7.2. Пусть в условиях следствия 7.1 либо 1) отображение $\nabla f_{S(x)}(x): X \rightarrow X^{*}$ непрерывно на любом отрезке внутри $\operatorname{dom} f$, либо 2) однозначное отображение $S(x)$ непрерьвно на любом отрезке внутри $\operatorname{dom} f$, и выполнено условие (1.6). Тогда функция $f(x)$ дифференцируема по Гато и $\nabla f(x)=\nabla f_{S(x)}(x)$ npu $x \in \operatorname{int} \operatorname{dom} f$. 
ДокАЗАТЕЛЬСТво. Если однозначное отображение $F(x)=\nabla f_{S(x)}(x)$ непрерывно на любом отрезке внутри $\operatorname{dom} f$, то выполнено условие б) следствия 7.1. Во втором случае выполнены условия теорем 3.1 и 2.2 , и правая часть формулы (3.1) опять состоит из одной точки $\nabla f_{S(x)}(x)$. Так как функция $f(x)$ непрерьвна, то она дифференцируема по Гато.

Пусть $X$ - гильбертово пространство. Напомним, что множество $S \subset X$ называется чебышевским, если для любой точки $x \in X$ сушествует ровно один элемент $s=s(x) \in S$ такой, что

$$
|s(x)-x|=\rho(x, S):=\inf _{s \in S}|s-x|, \quad x \in X .
$$

Отображение $s(x): X \rightarrow S$ назьвается метрической проекиией. Классический результат теории приближений таков: если метрическая проекция на чебышевское множество $S$ непрерьвна, то это множество выпукло.

Здесь мы докажем, что это утверждение верно при следующем ослабленном условии непрерывности: функция $x \rightarrow\langle s(x), \psi\rangle$ непрерывна по $x \in X$ на любом отрезке в $X$ при фиксированных $\psi \in X$. С этой целью рассмотрим функцию

$$
f(x):=\frac{|x|^{2}}{2}-\frac{\rho^{2}(x, S)}{2}=\sup _{s \in S}\left\{\langle s, x\rangle-\frac{|s|^{2}}{2}\right\} .
$$

Так как $S$ - чебышевское множество, то верхняя грань по $s \in S$ достигается ровно на одном элементе $s=s(x) \in S$. Поэтому согласно следствию 7.1 функция $f(x)$ дифференцируема по Гато. С учетом тождества (7.8) это справедливо и для функции $\rho^{2}(x, S)$. Из одного малоизвестного результата Л. П. Власова (см. [29, теорема 9$]$, а также [30, $§ 1.2])$ тогда следует, что чебышевское множество $S$ выпукло.

\section{§8. Вспомогательные результаты}

В формуле (3.6) часть субдифференциалов может быть пуста. В таких случаях необходимы критерии пустоты субдифференщиала.

Лемма 8.1. Если функиия $g(x): X \rightarrow \overline{\mathbb{R}}$ выпукла и непрерывна в точке $x_{1} \in X$, то следующие утверждения әквивалентны:

a) $\partial g\left(x_{0}\right)=\varnothing, \quad x_{0} \in \operatorname{dom} g$;

б) $x_{0} \notin \operatorname{int} \operatorname{dom} g u g^{\prime}\left(x_{0} ; x_{1}-x_{0}\right)=-\infty$;

в) $g^{\prime}\left(x_{0} ; x-x_{0}\right)=-\infty \quad \forall x \in \operatorname{int} \operatorname{dom} g$.

ДокАЗАТЕЛЬСТво. Если выпуклая функция $g(x)$ непрерывна в некоторой точке, то она не принимает значения $-\infty$, ее эффективное множество имеет непустую внутренность int dom $g$ и $\partial g(x) \neq \varnothing$ при $x \in \operatorname{int} \operatorname{dom} g$ [4]. Поэтому из условия а) следует, что $x_{0} \notin \operatorname{int} \operatorname{dom} g$. Так как по условию $x_{1} \in \operatorname{int} \operatorname{dom} g$, то $g^{\prime}\left(x_{0} ; \psi_{1}\right) \neq+\infty$, где $\psi_{1}:=x_{1}-x_{0}$. Если бы производная по направлениям $g^{\prime}\left(x_{0} ; \psi\right)$ была конечной в точке $\psi_{1}$, то она была бы непрерывна в этой точке (см. [4, c. 206]), а потому в ней и субдифференцируема:

$$
g^{\prime}\left(x_{0} ; x\right) \geqslant\left\langle x^{*}, x\right\rangle, \quad x \in X
$$


Следовательно, $x^{*} \in \partial g\left(x_{0}\right)$ (см. [14, с. 44]), т.е. $\partial g\left(x_{0}\right) \neq \varnothing$. Полученное противоречие показьвает, что $g^{\prime}\left(x_{0} ; \psi_{1}\right)=-\infty$. Таким образом, из а) следует б).

Чтобы увидеть, что из б) следует в), достаточно заметить, что при $x \in \operatorname{int} \operatorname{dom} g$ точка $\psi=x-x_{0}$ является внутренней в эффективном множестве вьпуклой функции $g^{\prime}\left(x_{0} ; \cdot\right)$, которая принимает значение $-\infty$ в точке $\psi_{1}($ см. $[14$, c. 36$])$. То, что из в) следует а), очевидно.

ЛЕмма 8.2. Если выполнены условия любой из теорем 2.1-2.5 и функции $f_{s}(x), s \in S$, непрерывны в некоторой точке $x_{1} \in \operatorname{dom} f$, то следующие утверждения эквивалентны:

a) $\partial f\left(x_{0}\right)=\varnothing, \quad x_{0} \in \operatorname{dom} f$;

б) $\partial f_{s}\left(x_{0}\right)=\varnothing \quad \forall s \in S\left(x_{0}\right)$;

в) $f_{s}^{\prime}\left(x_{0} ; x_{1}-x_{0}\right)=-\infty \quad \forall s \in S\left(x_{0}\right)$.

ДоКАЗАТЕЛЬСТво. В силу (3.4) из а) следует б), а по лемме 8.1 из б) следует в). Если же выполнено в), то по теоремам 2.1-2.5 имеем $f^{\prime}\left(x_{0} ; x_{1}-x_{0}\right)=-\infty$, так как $x_{1}-x_{0} \in K\left(x_{0} \mid \operatorname{dom} f\right)$. Очевидно, что тогда $\partial f\left(x_{0}\right)=\varnothing$, т.е. из в) следует а).

ЛЕмма 8.3. Для любой функции $g(x): X \rightarrow \overline{\mathbb{R}}$ при $x_{0} \in \operatorname{dom} g$

$$
\mathrm{O}^{+} \partial g\left(x_{0}\right)=N\left(x_{0} \mid \operatorname{dom} g\right) \text {. }
$$

ДокаЗАТЕЛЬСТво. В силу (1.5) $N\left(x_{0} \mid \operatorname{dom} g\right) \subset \mathrm{O}^{+} \partial g\left(x_{0}\right)$. Докажем обратное. Пусть $x_{0}^{*}+\lambda x^{*} \in \partial g\left(x_{0}\right)$ при всех $\lambda \geqslant 0$. Тогда

$$
g(x) \geqslant g\left(x_{0}\right)+\left\langle x_{0}^{*}+\lambda x^{*}, x-x_{0}\right\rangle \text { при всех } x \in X \text { и } \lambda \geqslant 0 .
$$

Поделив это неравенство на $\lambda>0$, при $\lambda \rightarrow+\infty$ получим $\left\langle x^{*}, x-x_{0}\right\rangle \leqslant 0 \forall x \in$ $\operatorname{dom} g$. Таким образом, если $x^{*} \in \mathrm{O}^{+} \partial g\left(x_{0}\right)$, то $x^{*} \in N\left(x_{0} \mid \operatorname{dom} g\right)$.

ЛЕмма 8.4. Если $A$ и $B$ - подмножества линейного пространства $X$, причем $B$ выпукло, то $\operatorname{conv}(A+B)=\operatorname{conv} A+B$.

Утверждение следует из того, что выпуклая оболочка множества состоит из конечных вьпуклых комбинаций его элементов.

ЛЕмма 8.5. Если функиии $f_{s}(x)$ вогнуты по $s$ на выпуклом множсестве $S\left(x_{0}\right)$ при всех $x \in X$, то будет выпукльм также и множество

$$
P:=\bigcup_{s \in S\left(x_{0}\right)} \partial f_{s}\left(x_{0}\right)
$$

ДокаЗАТЕЛЬСТво. Пусть $s_{i} \in S\left(x_{0}\right), x_{i}^{*} \in \partial f_{s_{i}}\left(x_{0}\right), \alpha_{i}>0, i=1,2$, $\alpha_{1}+\alpha_{2}=1, x \in X$. Тогда согласно (1.3) имеем

$$
f\left(s_{i}, x\right) \geqslant f\left(x_{0}\right)+\left\langle x_{i}^{*}, x-x_{0}\right\rangle, \quad i=1,2,
$$

откуда в силу вогнутости функции $f_{s}(x)$ по $s$ получим

$$
f\left(\alpha_{1} s_{1}+\alpha_{2} s_{2}, x\right) \geqslant \alpha_{1} f\left(s_{1}, x\right)+\alpha_{2} f\left(s_{2}, x\right) \geqslant f\left(x_{0}\right)+\left\langle\alpha_{1} x_{1}^{*}+\alpha_{2} x_{2}^{*}, x-x_{0}\right\rangle .
$$

Поскольку множество $S\left(x_{0}\right)$ вьпукло, то $s_{0}:=\alpha_{1} s_{1}+\alpha_{2} s_{2} \in S\left(x_{0}\right)$, т.е. $f\left(s_{0}, x_{0}\right)=$ $f\left(x_{0}\right)$. Тогда полученное неравенство означает, что $\alpha_{1} x_{1}^{*}+\alpha_{2} x_{2}^{*} \in \partial f_{s_{0}}\left(x_{0}\right)$, и, следовательно, множество $P$ вьпукло. 
ЛЕмма 8.6. Если функиии $f_{s}(x)$ пн. сверху по $s$ на компактном множестве $S\left(x_{0}\right)$ при всех $x \in X$, то мнохсество (8.1) слабо* замкнуто.

Доказательство аналогично доказательству теоремы об очистке (см. [4, с. 216]).

Лемма 8.7. Пусть $f_{s}(x): X \rightarrow \overline{\mathbb{R}}, s \in S$, - юббое семейство пн. снизу выпуклых функций таких, что множсество $S_{0}^{*}:=\left\{s \in S: f_{s}^{*}\left(x_{0}^{*}\right)=f^{*}\left(x_{0}^{*}\right)\right\}$ не пусто. Тогда следующие утверждения әквивалентны:

a) $x_{0} \in \partial f^{*}\left(x_{0}^{*}\right)$

б) существует әлемент $s \in S\left(x_{0}\right) \cap S_{0}^{*}$ такой, что $x_{0} \in \partial f_{s}^{*}\left(x_{0}^{*}\right)$.

ДоКАЗАТЕЛЬСТВо. Если $x_{0} \in \partial f_{s}^{*}\left(x_{0}^{*}\right)$ при $s \in S\left(x_{0}\right) \cap S_{0}^{*}$, то в силу полунепрерывности снизу функций $f_{s}(x)$ имеем

$$
\begin{gathered}
f_{s}\left(x_{0}\right)+f_{s}^{*}\left(x_{0}^{*}\right)=\left\langle x_{0}, x_{0}^{*}\right\rangle, \\
f\left(x_{0}\right)=f_{s}\left(x_{0}\right), \quad f^{*}\left(x_{0}^{*}\right)=f_{s}^{*}\left(x_{0}^{*}\right),
\end{gathered}
$$

что дает

$$
f\left(x_{0}\right)+f^{*}\left(x_{0}^{*}\right)=\left\langle x_{0}, x_{0}^{*}\right\rangle,
$$

т.е. $x_{0} \in \partial f^{*}\left(x_{0}^{*}\right)($ см. $[15$, теорема 23.5]).

Наоборот, если $x_{0} \in \partial f^{*}\left(x_{0}^{*}\right)$, то в силу полунепрерывности снизу функции $f(x)$ вьполнено (8.4). Так как $S_{0}^{*} \neq \varnothing$, то существует элемент $s \in S_{0}^{*}$ такой, что $f\left(x_{0}\right)+$ $f_{s}^{*}\left(x_{0}^{*}\right)=\left\langle x_{0}, x_{0}^{*}\right\rangle$. Поскольку $f\left(x_{0}\right) \geqslant f_{s}\left(x_{0}\right)$, то $f_{s}\left(x_{0}\right)+f_{s}^{*}\left(x_{s}^{*}\right) \leqslant\left\langle x_{0}, x_{0}^{*}\right\rangle$. Но это неравенство не может быть строгим. Поэтому выполнено (8.2) и $f\left(x_{0}\right)=f_{s}\left(x_{0}\right)$, т.е. $x_{0} \in \partial f_{s}^{*}\left(x_{0}^{*}\right)$ (по той же теореме 23.5 из [15]) и $s \in S\left(x_{0}\right)$.

ЛЕмма 8.8. Пусть $f_{s}(x), s \in S,-$ произвольное семейство функиий, $и$ пусть $x_{0}^{*} \in \partial f\left(x_{0}\right)$, причем

$$
\partial f\left(x_{0}\right)=\bigcup_{s \in S\left(x_{0}\right)} \partial f_{s}\left(x_{0}\right)
$$

(в частности, $S\left(x_{0}\right) \neq \varnothing$ ). Тогда $S_{0}^{*} \neq \varnothing u$

$$
f^{*}\left(x_{0}^{*}\right)=\min _{s \in S} f_{s}^{*}\left(x_{0}^{*}\right)
$$

ДокАЗАТЕЛЬСТво. Предположим противное. Поскольку $f(x) \geqslant f_{s}(x)$, то $f^{*}\left(x^{*}\right) \leqslant f_{s}^{*}\left(x^{*}\right), s \in S$, и, следовательно,

$$
f^{*}\left(x^{*}\right) \leqslant \inf _{s \in S} f_{s}^{*}\left(x^{*}\right)
$$

Тогда из предположения о пустоте множества $S_{0}^{*}$ вытекает, что

$$
f_{s}^{*}\left(x_{0}^{*}\right)>f^{*}\left(x_{0}^{*}\right) \quad \forall s \in S
$$


По теореме 23.5 из [15] включение $x_{0}^{*} \in \partial f\left(x_{0}\right)$ эквивалентно равенству (8.4). С учетом (8.8) поэтому получаем

$$
f\left(x_{0}\right)+f_{s}^{*}\left(x_{0}^{*}\right)>\left\langle x_{0}, x_{0}^{*}\right\rangle \quad \forall s \in S .
$$

Но при $s \in S\left(x_{0}\right)$ это неравенство означает, что

$$
f_{s}\left(x_{0}\right)+f_{s}^{*}\left(x_{0}^{*}\right)>\left\langle x_{0}, x_{0}^{*}\right\rangle
$$

т.е. по той же теореме $x_{0}^{*} \notin \partial f_{s}\left(x_{0}\right)$ для любого $s \in S\left(x_{0}\right)$. Таким образом, $x_{0}^{*} \notin \bigcup_{s \in S\left(x_{0}\right)} \partial f_{s}\left(x_{0}\right)$, что противоречит (8.5). Равенство (8.6) следует из (8.7) и непустоты множества $S_{0}^{*}$.

Автор признателен профессорам В. М. Тихомирову и Ж.-Б. Ириар-Уррути за помощь в этой работе.

\section{Список литературы}

1. Благодатских В.И. Теория дифференциальных включений. Ч. 1. М.: Изд-во МГУ, 1979.

2. Демьянов B. Ф., Рубинов А. М. Основы негладкого анализа и квазидифференциальное исчисление. М.: Наука, 1990.

3. Дубовичкий A.Я., Милютин A. A. Задачи на экстремум при наличии ограничений // ЖВМиМФ. 1965. Т. 5. №3. С. 395-453.

4. Иоффе А.Д., Тихомиров В. М. Теория экстремальных задач. М.: Наука, 1974.

5. Иоффе А.Д., Левин В. Л. Субдифференциалы выпуклых функций // Тр. Моск. мат. об-ва. 1972. Т. 26. С. 3-72.

6. Кларк $\Phi$. Оптимизация и негладкий анализ. М.: Наука, 1988.

7. Кусраев А.Г., Кутателадзе С. С. Субдифференциалы. Теория и приложения. Новосибирск: Наука, 1992.

8. Кутателадзе C. С. Основы функционального анализа. Новосибирск: Наука, 1983.

9. Левин В. Л. Применение теоремы Э. Хелли в выпуклом программировании, задачах наилучшего приближения и смежных вопросах // Матем. сб. 1969. Т. 79. № 2. С. 250-263.

10. Левин В. Л. Выпуклый анализ в пространствах измеримых функций. М.: Наука, 1985.

11. Левин В. Л. О некоторых свойствах опорных функционалов // Матем. заметки. 1968. T. 4. №6. С. $685-696$.

12. Минченко Л.И., Борисенко О.Ф. Дифференциальные свойства маргинальных функций и их приложения к задачам оптимизации. Минск: Наука и техника, 1992.

13. Обен Ж. П., Экланд И. Прикладной нелинейный анализ. М.: Мир, 1988.

14. Пшеничный Б. Н. Необходимые условия экстремума. 2-е изд. М.: Наука, 1982.

15. Рокафеллар Р. Т. Выпуклый анализ. М.: Наука, 1973.

16. Силин Д. В. Субдифференциалы выпуклых функций и интегралы от многозначных отображений // Вестн. Моск. ун-та. Сер. вычислит. матем. и кибернетика. 1984. № 1. C. $55-59$.

17. Соловьев В. Н. Двойственность невыпуклых экстремальных задач // ДАН СССР. 1990. T. 314. № 1. C. $135-138$.

18. Соловъев В. Н. О субдифференциале максимума семейства выпуклых функций // УМН. 1996. T. 51. № 2. C. $175-176$.

19. Соловьев В.Н. О субдифференциале максимума выпуклых функций // Докл. РАН. 1997. T. 357. № 1. C. 24, 25.

20. Сухарев А.Г., Тимохов А.В., Федоров В.В. Курс методов оптимизации. М.: Наука, 1986. 
21. Экланд И., Темам Р. Выпуклый анализ и вариационные проблемы. М.: Мир, 1979.

22. McFadden D. Production Economics, a Dual Approach to Theory and Applications. V. 1. Amsterdam: North-Holland, 1978.

23. Benoist J., Hiriart-Urruty J.-B. What is the subdifferential of the closed convex hull of a function? // SIAM J. on Math. Analysis (to appear).

24. Hiriart-Urruty J.-B., Moussaoui M., Seeger A., Volle M. Subdifferential calculus without qualification conditions, using approximate subdifferentials: a survey // Non-linear Analysis, Theory, Methods and Applications. 1995. V. 24. № 12. P. 1727-1754.

25. Soloviov V. Duality for Nonconvex Optimization and Its Applications // Anal. Math. 1993. V. 19. № 4. P. 297-315.

26. Valadier $M$. Sous differentiel d'une borne superieur et d'une somme continue de fonctions convexes // C. R. Acad. Sci. Paris. 1969. V. 268. P. 39-42.

27. Volle M. Sous-differetiel d'une enveloppe superieure de fonctions convexes // C. R. Acad. Sci. Paris. 1993. V. 317. Ser. I. P. 845-849.

28. Volle $M$. Calculus rules for global approximate minima and applications to approximate subdifferential calculus // J. of Global Optimization. 1994. V. 5. P. 131-157.

29. Власов Л. П. Несколько теорем о чебышевских множествах // Матем. заметки. 1972. T. 11. № 2. C. 135-144.

30. Соловьев B. H. Двойственные экстремальные задачи и их применения к задачам минимаксного оценивания // УМН. 1997. Т. 52. №4. С. 49-86.

Поступило в редакцию

9.IX.1996 